\title{
Broadband Wireless Channel in Composite High-Speed Railway Scenario: Measurements, Simulation, and Analysis
}

\author{
Jianwen Ding, ${ }^{1,2}$ Lei Zhang, ${ }^{3}$ Jingya Yang, ${ }^{4}$ Bin Sun,, ${ }^{1,2}$ and Jiying Huang ${ }^{5}$ \\ ${ }^{1}$ National Research Center of Railway Safety Assessment, Beijing Jiaotong University, Beijing 100044, China \\ ${ }^{2}$ Beijing Engineering Research Center of High-Speed Railway Broadband Mobile Communications, Beijing Jiaotong University, \\ Beijing 100044, China \\ ${ }^{3}$ Shanghai Research Center for Wireless Communications, Shanghai 201210, China \\ ${ }^{4}$ State Key Laboratory of Rail Traffic Control and Safety, Beijing Jiaotong University, Beijing 100044, China \\ ${ }^{5}$ School of Electronic and Information Engineering, Beijing Jiaotong University, Beijing 100044, China \\ Correspondence should be addressed to Jianwen Ding; jwding@bjtu.edu.cn
}

Received 30 May 2017; Revised 2 September 2017; Accepted 22 October 2017; Published 15 November 2017

Academic Editor: Dajana Cassioli

Copyright ( 2017 Jianwen Ding et al. This is an open access article distributed under the Creative Commons Attribution License, which permits unrestricted use, distribution, and reproduction in any medium, provided the original work is properly cited.

\begin{abstract}
The rapid development of high-speed railway (HSR) and train-ground communications with high reliability, safety, and capacity promotes the evolution of railway dedicated mobile communication systems from Global System for Mobile CommunicationsRailway (GSM-R) to Long Term Evolution-Railway (LTE-R). The main challenges for LTE-R network planning are the rapidly timevarying channel and high mobility, because HSR lines consist of a variety of complex terrains, especially the composite scenarios where tunnels, cuttings, and viaducts are connected together within a short distance. Existing researches mainly focus on the path loss and delay spread for the individual HSR scenarios. In this paper, the broadband measurements are performed using a channel sounder at $950 \mathrm{MHz}$ and $2150 \mathrm{MHz}$ in a typical HSR composite scenario. Based on the measurements, the pivotal characteristics are analyzed for path loss exponent, power delay profile, and tap delay line model. Then, the deterministic channel model in which the $3 \mathrm{D}$ ray-tracing algorithm is applied in the composite scenario is presented and validated by the measurement data. Based on the ray-tracing simulations, statistical analysis of channel characteristics in delay and Doppler domain is carried out for the HSR composite scenario. The research results can be useful for radio interface design and optimization of LTE-R system.
\end{abstract}

\section{Introduction}

Over the past few years, the world has witnessed the rapid development of the high-speed railways (HSRs) in China. The GSM-R system, which is specially designed and standardized for communication between train and control centers, could provide voice group call, functional addressing, location dependent addressing, enhanced multilevel priority and preemption, train control safety data transmission, and dispatching non-safety data transmission service. However, the GSM-R still has some limitations to meet a diversity of data transmission including security monitoring and maintenance information in real network operation since it is only available to narrowband communications. Thus, the broadband wireless train-ground communication on HSR plays an important role in train operation control, monitoring, and maintenance data transmission and makes a great number of applications possible $[1,2]$. To satisfy the increasing demand for railway services, HSR faces the challenge that is to deploy broadband communication system called LTE-R [3-6]. The LTE-R has been identified as the technical direction of the next generation of railway mobile communication in HSR environments due to its technical advantages, such as increased performance, flexible quality of service (QoS) profile, supporting mission critical service, and high-speed mobility [7, 8]. Currently, a series of studies on HSR broadband communication system are being carried out, while radio propagation is the main research hotspot because the high performance of communication system relies on excellent wireless network coverage. The sustainable development of railway broadband mobile communication system needs to keep abreast of HSR wireless channel 
characteristics $[9,10]$. Establishing reliable and credible HSR channel model is the precondition to realize the efficient, reliable, and safe data transmission under the conditions of high mobility and high data rate.

The researches on broadband and narrowband channels in HSR or subway environments have been conducted over the last few years. The small-scale fading behavior, which is defined as signal variations occurring within very short distances, has been discussed in large numbers of papers for broadband mobile communications. Several wireless channel propagation measurement activities have been performed in different kinds of HSR scenarios, such as the train station [11-13], the viaduct [14-17], the cutting [18, 19], the crossing bridge [20], the railway tunnel [21], the subway tunnel [22, 23], and the passageway tunnel [24]. The empirical path loss model and small-scale fading parameters have been obtained at $930 \mathrm{MHz}$. The propagation characteristics in a small passageway tunnel for non-line-of-sight (NLoS) are studied in [24], and the model is given to predict the path loss, power delay profile (PDP), root-mean-square (RMS) delay spread, and coherence bandwidth. The tap delay line (TDL) models and Doppler characterizations for HSR hilly scenario are put forward in [25]. In [26], the propagation mechanisms at $2.35 \mathrm{GHz}$ have been investigated for the viaduct scenario, where the path loss, time delay spread, and $\mathrm{K}$-factor have been obtained to establish the statistical position-based channel models. In [27], a cooperative antenna system is built on separated train carriages and a Doppler frequency offset estimation and joint channel estimation algorithm exploited multiantenna diversity gains are put forward. These studies mainly concentrate on channel parameters in an individual scenario. However, as a result of the high-speed mobility in the actual HSR environment, scenarios including the tunnel, cutting, and viaduct are continuous for wireless communication. Thus, the mobile station will experience many kinds of scenarios in a short period of time, and these scenarios are inseparable for the mobile station. Call drops and data transmission errors occur in such composite scenarios frequently. In summary, existing studies generally ignore the quick transition from one scenario to another. Thus, the channel model for a composite scenario is still inadequate and the nonstationary characteristic of time-varying and fastfading channels in HSR composite scenario still needs further investigation.

The deterministic modeling technique based on raytracing (RT) simulation is also an important method to model HSR channels [28, 29]. This method takes into account the direct path, reflections, and scattering and models the propagation channel in a specific scenario using the morphological and geographical information. However, in a complicated scenario, the RT method requires a large amount of computations. In general, for the sake of reducing the computational time in the complicated scenarios, only the first- and secondorder reflections are taken into consideration. The output of the deterministic channel models for each link is the timevarying channel impulse response (CIR) which characterizes the frequency-selective channel. The 3-dimensional (3D) scenario reconstruction is the basis of the RT modeling, which has been studied in the literature, for example, [30, 31].
The main contributions of this paper lie in the following aspects:

(1) A broadband wireless channel measurement campaign is conducted in HSR composite scenario by using the channel sounding system at the frequencies of $950 \mathrm{MHz}$ and $2150 \mathrm{MHz}$.

(2) Based on the measurement data, we explicate the phenomenon of the variation trends of path loss, PDP, mean excess delay, and the RMS delay spread in 6 typical areas of the composite scenario. Then, the TDL based channel models are formed, which are helpful for the characterization of the HSR timevarying channel.

(3) The HSR composite scenario is reconstructed and the 3D RT simulator is validated by the measurement data. Then, the small-scale fading, PDP, Doppler statistical properties, and the correlation coefficients between delay and Doppler domain are investigated. This is helpful for the development of HSR broadband communication system in the foreseeable future.

The rest of this paper is organized as follows. The measurement scenario, channel sounder configuration, and measurement campaign are provided in Section 2. Section 3 presents the measurement results with statistical analysis, and a novel TDL channel model for HSR composite scenarios is established. Section 4 provides the 3D scenario reconstruction of the composite HSR scenario; a 3D rayoptical based channel simulation and stochastic analysis are introduced as well. In Section 5, the deterministic modeling of the channel in HSR composite scenario and the statistical modeling from the measured data in the same scenario are compared and validated. Moreover, the small-scale fading, Doppler statistical properties, and correlation coefficients between delay and Doppler domain are analyzed in detail. Finally, the conclusions are drawn in Section 6.

\section{HSR Channel Measurements}

2.1. Measurement System Configuration. The measurement system consists of a channel sounder including transmitter (Tx) and receiver (Rx), oscilloscope, and antennas. The measurement specifications are described in detail below.

2.1.1. Channel Sounder. A channel sounder serves as a systematic device to measure the behaviors of wireless signals in the specific environment [32]. The test platform adopts the narrow pulse technology and can be used for the broadband channel sounding, with the parameters listed in Table 1. The $\mathrm{Tx}$ is composed of a broadband frequency synthesizer, pulse modulator, pulse generator, and broadband power amplifier. The synthesizer is controlled by the customized software on $\mathrm{PC}$ to program the transmission frequency. The transmitted signal is modulated by pulse modulator to get a narrow pulse with selectable pulse widths. The high quality pulse generator is built to synchronize with the clock signal. Eventually, a high power amplifier is used to amplify the modulated sounding signal. The receiver is composed of local oscillator, low noise 
TABLE 1: Channel sounder parameters.

\begin{tabular}{|c|c|}
\hline Parameters & Settings \\
\hline \multicolumn{2}{|l|}{ Transmitter } \\
\hline Frequency range & $35 \mathrm{MHz}-3000 \mathrm{MHz}$ \\
\hline Output power & $35 \mathrm{dBm}$ \\
\hline Pulse width & $30 \mathrm{~ns}, 45 \mathrm{~ns}$, and $60 \mathrm{~ns}$ \\
\hline Pulse period & 1 us \\
\hline Rise and fall time & $3 \mathrm{~ns}$ \\
\hline Intermediate frequency (IF) bandwidth & $100 \mathrm{MHz}$ \\
\hline \multicolumn{2}{|l|}{ Receiver } \\
\hline Frequency range & $100 \mathrm{MHz}-4550 \mathrm{MHz}$ \\
\hline IF & $150 \mathrm{MHz}$ \\
\hline IF bandwidth & $100 \mathrm{MHz}$ \\
\hline Sensitivity & $-100 \mathrm{dBm}$ \\
\hline Noise figure & $3 \mathrm{~dB}$ \\
\hline Video output & $1.2 \mathrm{Vpp}$ \\
\hline
\end{tabular}

amplifier (LNA), mixer, intermediate frequency filters, and logarithmic amplifier used as the demodulator. The received signal is sent to the LNA. Then, the amplified signal is mixed with a phase-locked loop (PLL) for superhet receiving to get a $+/-150 \mathrm{MHz}$ intermediate frequency, through an addition or subtraction to the transmitted signal frequency. Then, a $100 \mathrm{MHz}$ band-pass filter is set to filter out unwanted parts. Finally, the logarithmic amplifier is used as an envelope detector, which connects to the oscilloscope to provide the magnitude of the received signal [33].

2.1.2. Oscilloscope. The output demodulated signal of the receiver is sent to the digital oscilloscope, which allows capturing digitized PDP in real time and saving data on the hard disk. In order to meet the requirements of high sampling rate, Infiniium MSO9104A from Keysight is used, which offers a bandwidth up to $1 \mathrm{GHz}$ and channels with responsive deep memory to ensure superior viewing of signals under test.

2.1.3. Antennas. The channel sounder is equipped with the L-Com HG72714P-090 panel directional antenna whose polarization is vertical, with $17^{\circ}$ vertical and $90^{\circ}$ horizontal beamwidth, as shown in Figure 1(a) and Table 2. The Rx antenna is omnidirectional with 3 meters height plus the bracket. The Rx antenna is L-Com HG72107U, which is also vertically polarized and has the gain of $4 \mathrm{dBi}$ and $7 \mathrm{dBi}$ in different bands, as shown in Figure 1(b) and Table 2.

2.2. Measurement Scenario. In the construction of railway in mountainous region, the cuttings are often used to help the HSR cross the large obstacles. A standard deep cutting with the slopes of both sides having the similar inclination can produce abundant reflection and scattering components. Viaducts with a height of $10 \mathrm{~m}$ to $30 \mathrm{~m}$ are also used in HSR to ensure flatness and straightness of rails. The height of the antenna on the roof of the train is increased due to viaduct, and this reduces the number of scatterers and forms a
TABLE 2: Tx and Rx antenna parameters.

\begin{tabular}{lc}
\hline Parameters & Settings \\
\hline Frequency range & Tx antenna \\
Gain & $698-960 \mathrm{MHz}, 1710-2700 \mathrm{MHz}$ \\
Vertical beamwidth & $13 \mathrm{dBi}, 14 \mathrm{dBi}$ \\
Horizontal beamwidth & $17^{\circ}$ \\
Polarization & $90^{\circ}$ \\
Front to back ratio & Vertical \\
Dimensions & $\geq 23 \mathrm{~dB}$ \\
& $1568 \times 320 \times 160 \mathrm{~mm}$ \\
Frequency range & \\
Gain & $698-960 \mathrm{MHz}, 1710-2700 \mathrm{MHz}$ \\
Vertical beamwidth & $4 \mathrm{dBi}, 7 \mathrm{dBi}$ \\
Horizontal beamwidth & $55^{\circ} 28^{\circ}$ \\
Polarization & $360^{\circ}$ \\
Lengths & Vertical \\
\hline
\end{tabular}

relatively "clear" line-of-sight (LoS) propagation channel. Therefore, cutting and viaduct have a pronounced effect on channel propagation characteristics, which provides the motivation for investigating the composite scenario of cutting and viaduct in this paper.

The broadband measurements were performed in Xinzhou section of the "Datong-Xian" HSR in China, as shown in Figure 2. A tunnel with the height of up to $9 \mathrm{~m}$, a cutting of $130 \mathrm{~m}$ length, a viaduct of $280 \mathrm{~m}$ length, and a cutting of $100 \mathrm{~m}$ length are linked end to end. In this case, it is more likely to receive multipath components with a higher height of the upper cutting than that of the receiving antenna. The numerous multipath components caused by the side walls result in serious fading. Moreover, a semiclosed space is generated by the structure of the cutting and the mountain, which may contain a large number of scattering and reflection components.

2.3. Measurement Campaign. This paper mainly analyzes and discusses the effects of multipath components in composite scenarios. In order to achieve this goal, a series of broadband measurements at $950 \mathrm{MHz}$ and $2150 \mathrm{MHz}$ bands which may be used for LTE-R in future were conducted. The channel sounder transmitter was installed on the platform at the top of the cutting, the Tx antenna was $35 \mathrm{~m}$ high from the rail surface and $20 \mathrm{~m}$ away from the tunnel portal. The pitch angle of transmitting antenna was about $5^{\circ}$. The channel sounder receiver with antenna moved along the track, and measurement data were collected at 50 different locations.

The measurement environment can be classified as four regions in the composite scenario: locations 1 and 2 belong to region $\mathrm{I}$, that is, the tunnel portal; locations $3-15$ fall into region II which is cutting 1; locations 16-44 belong to region III, where a viaduct exists, and region IV, which is cutting 2 , includes the rest of the locations. The sampling interval is $50 \mathrm{~ns}$ and approximately 20000 CIRs for each snapshot are collected. Each neighboring snapshot has a distance of $10 \mathrm{~m}$. 

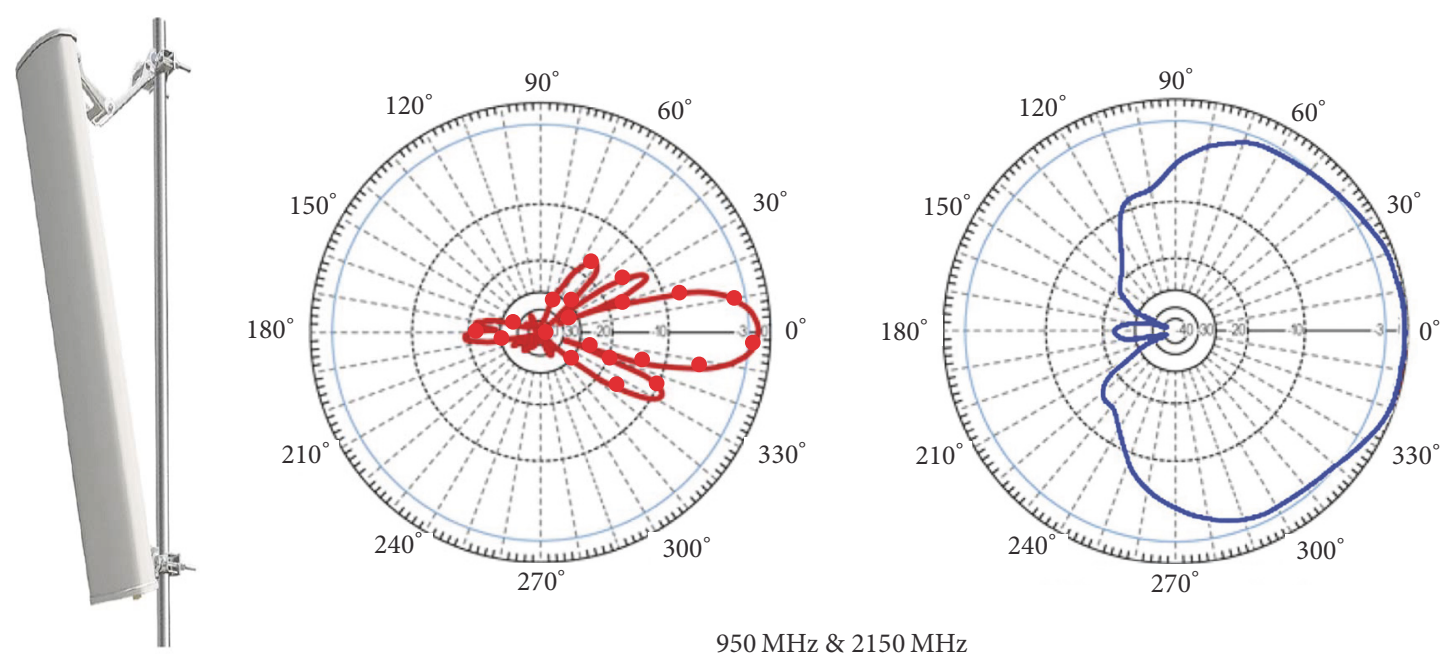

$950 \mathrm{MHz} \& 2150 \mathrm{MHz}$

$$
\underset{\text { — Horizontal }}{\longrightarrow \text { Vertical }}
$$

(a) Tx antenna pattern

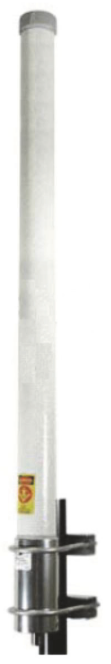

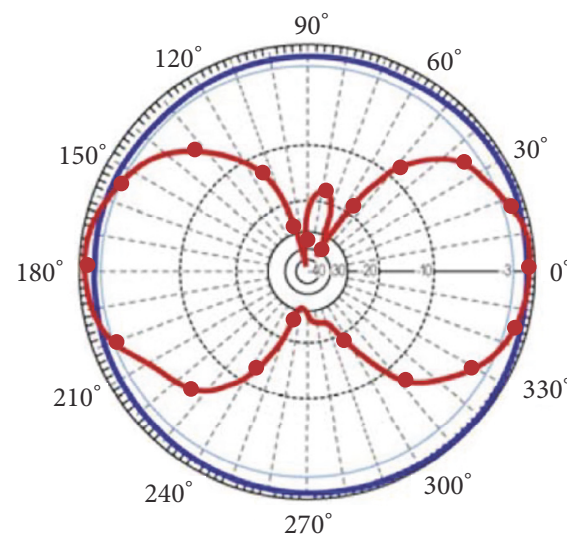

$950 \mathrm{MHz}$

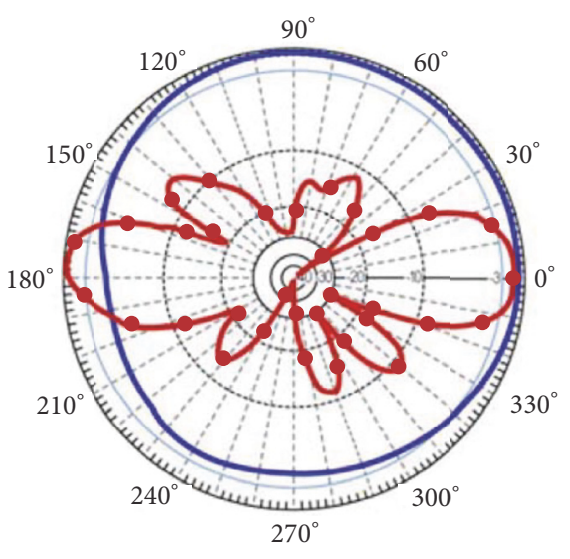

$2150 \mathrm{MHz}$
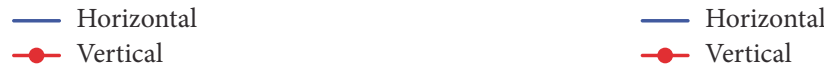

(b) Rx antenna pattern

Figure 1: Antenna pattern.

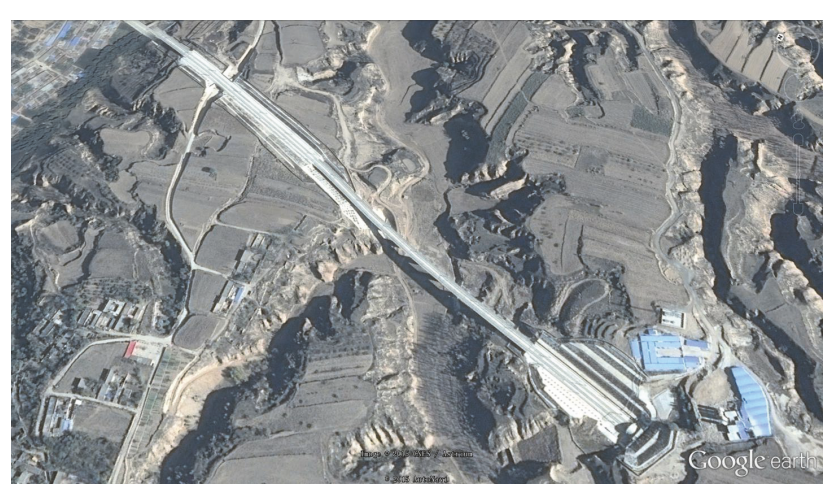

(a) Scenario from Google Earth

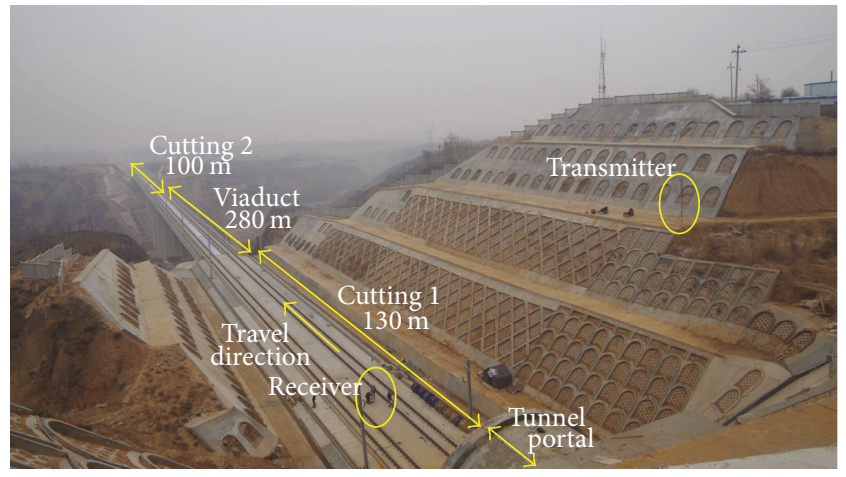

(b) Field measurement scenario

FIgure 2: The measured composite scenario in Datong-Xi'an HSR. 


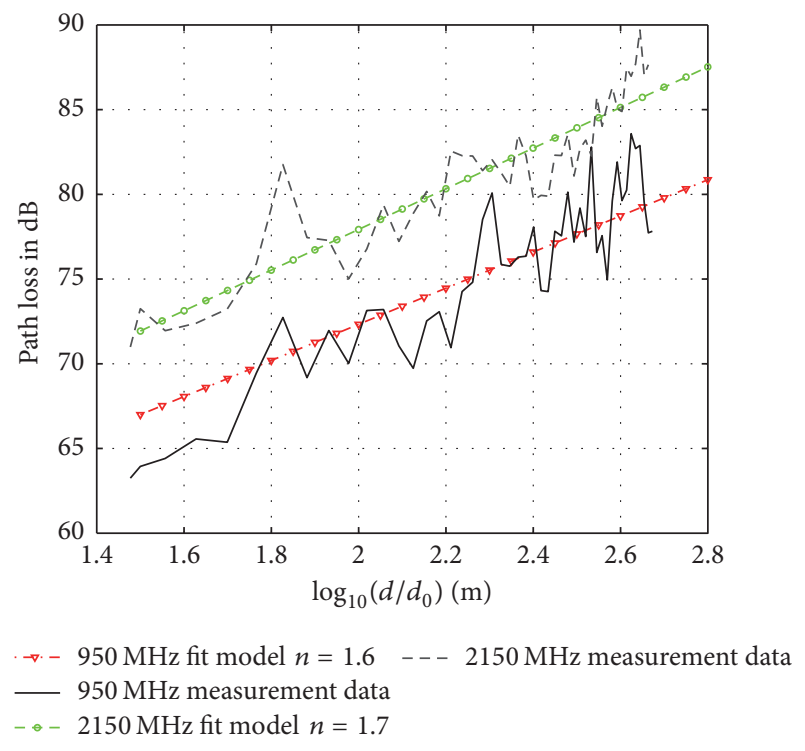

Figure 3: Path loss at $950 \mathrm{MHz}$ and $2150 \mathrm{MHz}$ in the composite scenario.

\section{Measurements Results and Analysis}

The knowledge of wireless channel fading behavior is helpful for designing fading countermeasures. Thus, parameterizing the fading properties and developing channel model for HSR are necessary. This section will make a detailed analysis of the measurement results.

3.1. Path Loss Analysis. The path loss model assumes a linear dependency between the path loss in $\mathrm{dB}$ and the logarithm of distance $d[34]$, shown as

$$
\mathrm{PL}[\mathrm{dB}](d)=\mathrm{PL}_{\mathrm{FS}}\left(d_{0}\right)+10 \bar{n} \log _{10}\left(\frac{d}{d_{0}}\right)+X_{\sigma},
$$

where $\mathrm{PL}_{\mathrm{FS}}\left(d_{0}\right)$ is the frequency-dependent free space path loss at the reference distance $d_{0}, \bar{n}$ is the average path loss exponent (PLE) over distance, and $X_{\sigma}$ is a zero-mean Gaussian distributed random variable with standard deviation $\sigma$ describing the random shadowing. We find that the $d_{0}=1 \mathrm{~m}$ close-in (CI) free space reference path loss model is a simple physically based one-parameter PLE model $[35,36]$, which is more stable across frequencies and environments than the traditional floating-intercept (FI) least-squares regression equation line. The fixed-intercept is given as

$$
\mathrm{PL}_{\mathrm{FS}}\left(d_{0}\right)=20 \log _{10}\left(\frac{4 \pi d_{0}}{\lambda}\right)
$$

where $d_{0}=1$ and $\lambda$ is the carrier wavelength. The CI models for the two carrier frequencies of $950 \mathrm{MHz}$ and $2150 \mathrm{MHz}$ are shown in Figure 3 with the measured data. As can be seen from Figure 3, the PLE of the composite scenario is 1.6 and 1.7 at $950 \mathrm{MHz}$ and $2150 \mathrm{MHz}$, respectively. Such small PLE values imply the wave-guide effect in the propagation of this composite scenario. Apart from the direct path between the Tx and the Rx, numerous reflected components caused by the

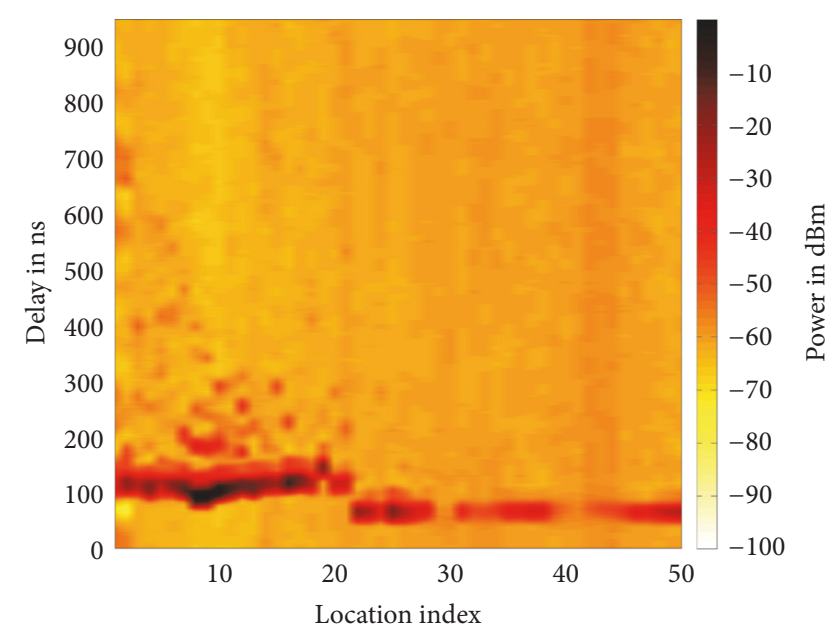

Figure 4: PDP at $950 \mathrm{MHz}$ in the composite scenario.

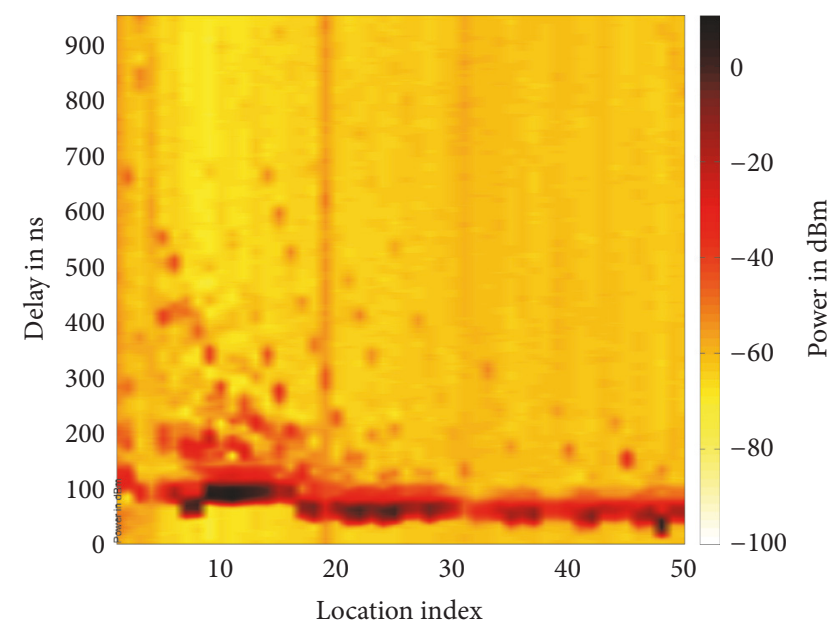

FIGURE 5: PDP at $2150 \mathrm{MHz}$ in the composite scenario.

cutting slopes and mountains also enforce the propagation, and, therefore, their superposition decreases the PLE values. Similar observation at $930 \mathrm{MHz}$ in HSR viaduct scenarios has been reported in [37], in which the PLEs are between 1.5 and 1.7.

3.2. Power Delay Profile (PDP). The PDP is widely used for modeling multipath fading channel. The extent of multipath effects on radio channel can be clearly determined by the time delay spread. Channel parameters such as the first and second significant multipath components can be extracted from the PDP curve.

Since the HSR base station is always installed on one side of the track and the antenna is higher than the track by at least $25 \mathrm{~m}$ except for tunnel part, railway environment is a typical scenario with LoS propagation. Usually, the radio wave coming from LoS path offers the dominant component, and the radio waves coming from other directions are scattered or reflected by the steep walls or hills, causing the time delay spread.

From Figures 4 and 5, it is found that the PDP fluctuates in this composite scenario along the track at the frequency 
TABLE 3: Parameters of time dispersion at two frequencies.

\begin{tabular}{|c|c|c|c|c|c|}
\hline \multicolumn{2}{|c|}{ Region index } & I & II & III & IV \\
\hline \multicolumn{2}{|c|}{ Corresponding scenario } & Tunnel & Cutting & Viaduct & Cutting \\
\hline $950 \mathrm{MHz}$ & Mean excess delay (ns) & 374.66 & 193.11 & 60.61 & 31.40 \\
\hline $950 \mathrm{MHz}$ & Average RMS delay spread (ns) & 218.67 & 125.40 & 50.01 & 40.20 \\
\hline $950 \mathrm{MHz}$ & Average number of multipaths & 6 & 5 & $1 \sim 2$ & $1 \sim 2$ \\
\hline $2150 \mathrm{MHz}$ & Mean excess delay (ns) & 270.68 & 195.68 & 66.31 & 43.62 \\
\hline $2150 \mathrm{MHz}$ & Average RMS delay spread (ns) & 120.44 & 135.18 & 52.03 & 38.81 \\
\hline $2150 \mathrm{MHz}$ & Average number of multipaths & 4 & 5 & $1 \sim 3$ & $1 \sim 2$ \\
\hline
\end{tabular}

of $950 \mathrm{MHz}$ and $2150 \mathrm{MHz}$. Generally, waves at these two frequencies have quite similar behaviors, with the distance changes [33]. In the first $150 \mathrm{~m}$, the propagation at both 950 $\mathrm{MHz}$ and $2150 \mathrm{MHz}$ is with quite clear delay spread. More significant delay spread is demonstrated when $\mathrm{Rx}$ is closer to the mountain tunnel, which is due to the strong reflection from the wall above the tunnel. Then, the delay spread has the tendency to decrease gradually when the Rx moves to the cutting, which is due to the open space. However, there are still rich multipath components. The delay spread decreases when the $\mathrm{Rx}$ is close to $150 \mathrm{~m}$ from the Tx. This point is exactly the entrance of viaduct in the open area. Thus, a number of the multipath components disappear, and the propagation is dominated by the direct ray.

The typical PDPs on different scenarios are demonstrated in Figures 6(a)-6(f), respectively. Upon the $\mathrm{Rx}$ being in region $I$, that is, the tunnel portal, due to the direct path to the Tx being blocked by the tunnel hall in the shadowed area, it is the NLoS scenario. Radio propagation at both frequencies shows longer and clearer time delays in the NLoS area. In particular, the delay spread at $950 \mathrm{MHz}$ is longer and the severe multipath effect is reflected, compared with $2150 \mathrm{MHz}$. Upon the Rx being in the region II, there are obvious LoS component and multipath components caused by the cutting steep walls. Upon the $\mathrm{Rx}$ being in region III, which is the viaduct scenario, it is difficult to detect the multipath components due to the disappearance of reflector. The contribution of multipath components from distant mountain scattering can also be ignored. The delay spread greatly decreases. Upon the $\mathrm{Rx}$ being in region IV, which is the cutting 2 scenario, there is only the direct path because the cutting height is low and the excess delay of reflected signals is close to that of the dominant signal. The power of reflected signal originated by the remote cutting is also small, and, therefore, a clear path cannot be formed.

3.3. Analysis of Time Dispersion. As a general method for analyzing broadband channel measurements, the PDP can be quantified by the mean excess delay and RMS delay spread, which describe the time dispersion characteristics of the channel in a specific scenario. Delay of the multipath component is estimated relatively to the first detectable peak path $[38,39]$. The mean excess delay $\bar{\tau}$ is the first moment of the PDP and is defined as

$$
\bar{\tau}=\frac{\sum_{k=1}^{K} P\left(\tau_{k}\right) \tau_{k}}{\sum_{k=1}^{K} P\left(\tau_{k}\right)},
$$

where $P\left(\tau_{k}\right)$ is the power level of the $k$ th path and $\tau_{k}$ is the relative time delay of the $k$ th path. The commonly quantified RMS delay spread $\sigma_{\tau}$ is the square root of the second central moment of the PDP and is presented in (4), which can be used to extract the number of channel taps [40].

$$
\sigma_{\tau}=\sqrt{\overline{\tau^{2}}-(\bar{\tau})^{2}}
$$

In general, the mean excess delay and the RMS delay spread rely on the selection of noise threshold, which is used to distinguish the received multipath components power from the thermal noise power for raw PDPs. In this paper, the noise threshold is set to be $6 \mathrm{~dB}$ above the noise level of PDP, as given in $[41,42]$. Thus, the peak that is greater than the threshold is considered as a resolvable path. Meanwhile, to reduce the implementation complexity of the channel model, the number of channel taps should be specified. Using RMS delay spread to determine the number of taps $L$ is given by [38]

$$
L=\left[\frac{\max \left(\sigma_{\tau}\right)}{T_{c}}\right]+1,
$$

where $\sigma_{\tau}$ is the RMS delay spread and $T_{c}$ is the pulse duration, which is $30 \mathrm{~ns}$ in this paper.

In Table 3, the average number of multipath components, the mean excess delay, and RMS delay spread are calculated. The RMS delay spreads at the two frequencies are illustrated in Figure 7. The values of RMS delay spreads for region III and region IV lie in the range of $0-100 \mathrm{~ns}$. This is due to the presence of the dominant LoS component, with almost no more other distinguishable multipath components. For the region close to the cutting area, RMS delay spread becomes larger, whereas the RMS delay spread is mainly in the range of 100-300 ns in the cutting scenario, which reflects the severe fading effects. In this area, there are approximately 5-6 multipath components due to the fact that there are more reflection and scattering components from the cutting wall and remote hills. The RMS delay spread values of different frequencies are close to each other. Note that similar results about the delay statistics are shown in [43] for the approximatively $950 \mathrm{MHz}$ and $2150 \mathrm{MHz}$ cases.

3.4. TDL Channel Model. The TDL based channel model is helpful for analyzing the time-varying channel [26]. The TDL based models are developed according to the hypothesis; namely, the distinguishable channel taps are discrete and have different delays in HSR composite scenario. In this subsection, the paper parameterizes the tap number, the relative 


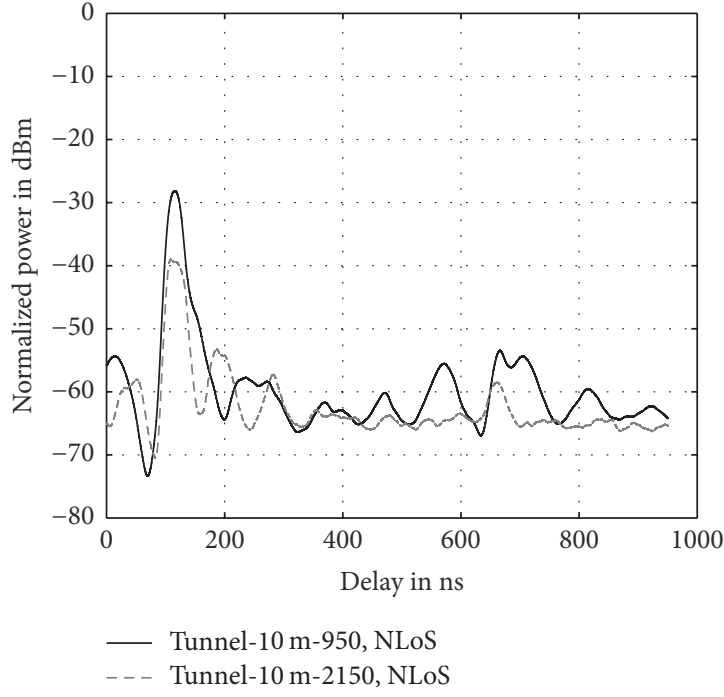

(a) Tunnel portal

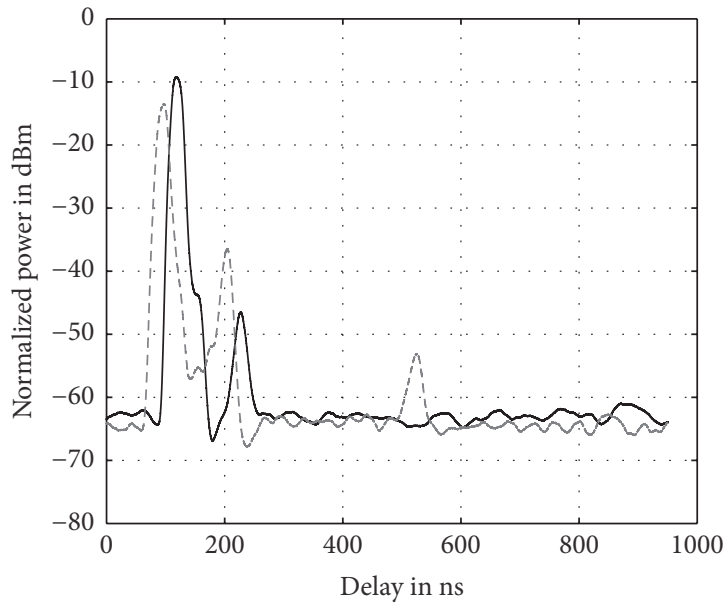

_ Viaduct near from cutting 1-950, LoS

- - - Viaduct near from cutting 1-2150, LoS

(c) Viaduct near cutting 1

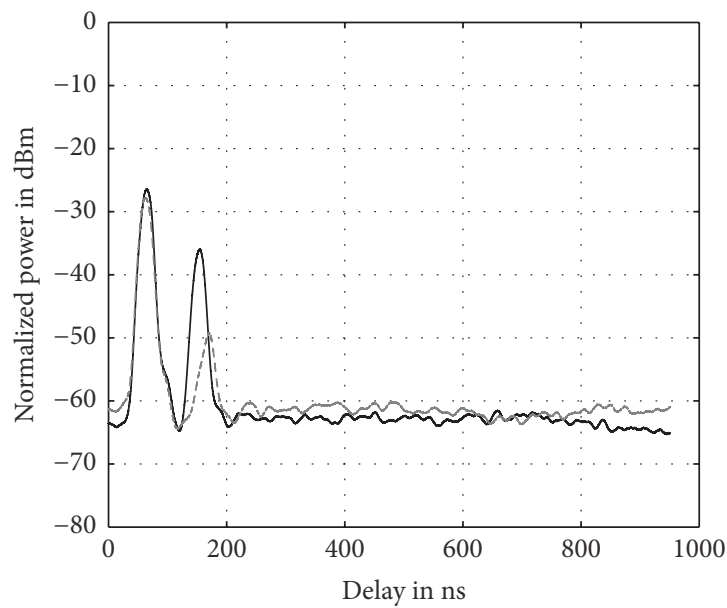

_ Viaduct near from cutting 2-950, LoS

- - - Viaduct near from cutting 2-2150, LoS

(e) Viaduct near cutting 2

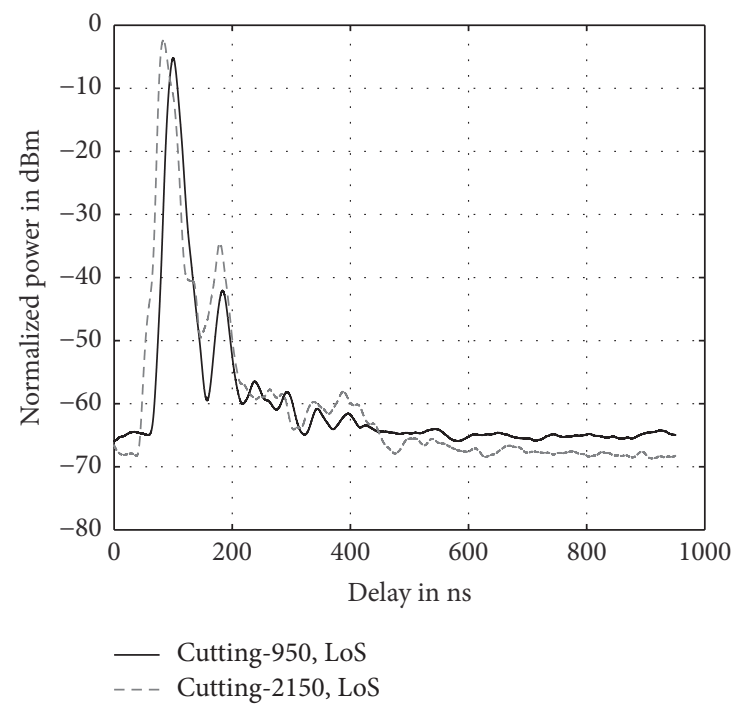

(b) Cutting 1

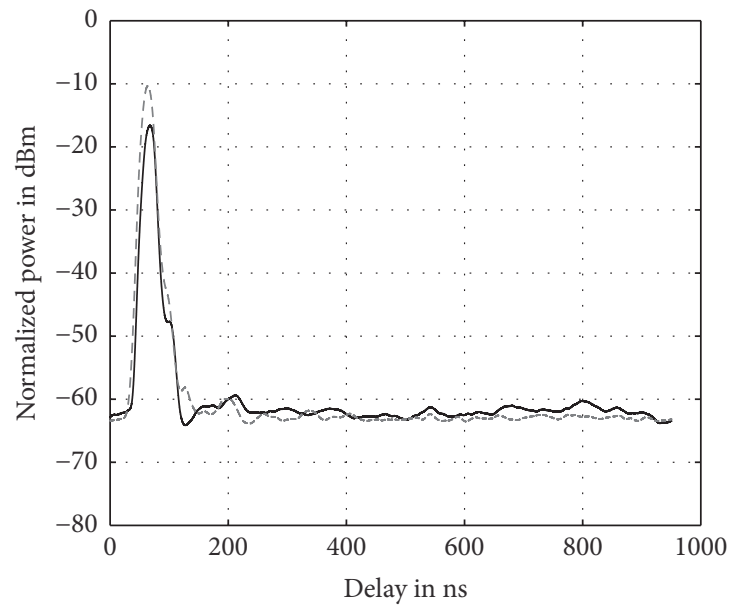

- Viaduct-950, LoS

- _ - Viaduct-2150, LoS

(d) Viaduct

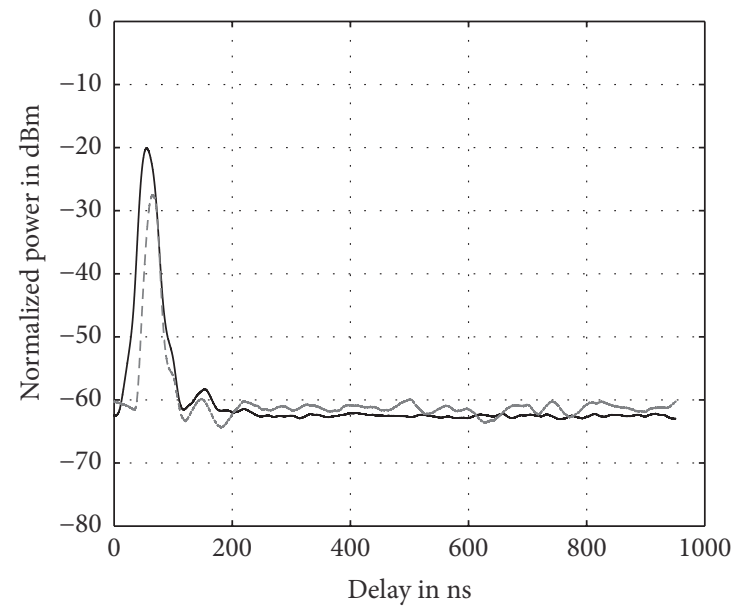

Cutting2-950, LoS

Cutting2-2150, LoS

(f) Cutting 2

FIGURE 6: Typical PDP for composite HSR scenario. 


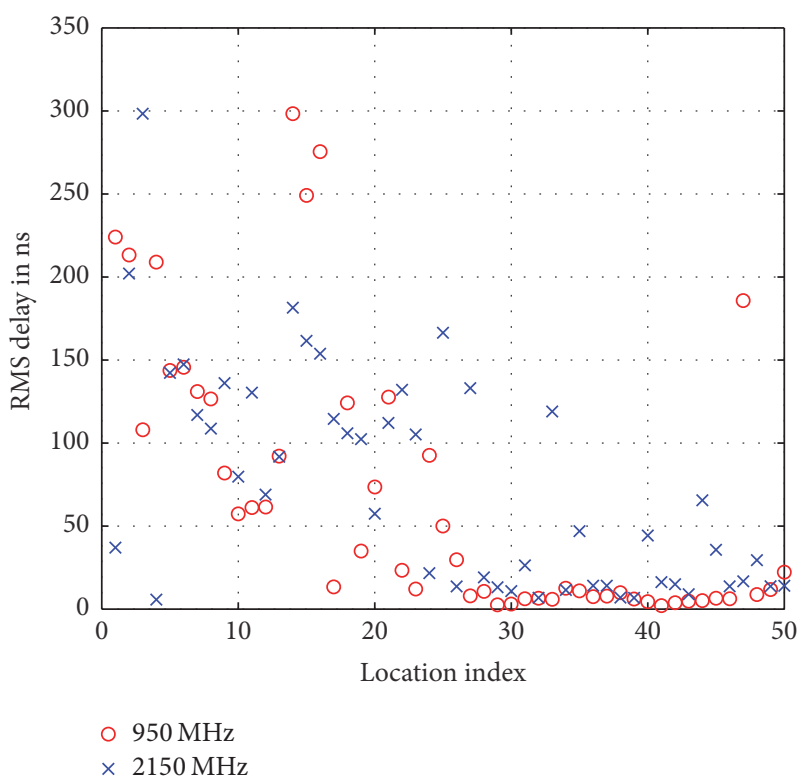

FIGURE 7: RMS delay spread at two frequencies.

delay spread, and the average gain in different scenarios on the basis of a detailed analysis of the measurement data.

3.4.1. TDL Channel Model under Tunnel Portal. Table 4 puts forward the TDL channel model under tunnel portal scenario with $\mathrm{Tx}$ on the roof of the cutting near the tunnel. It can be concluded that the number of multipath components is large, and the tunnel portal is very consistent with the NLoS scenario. The $2.4 \mathrm{GHz}$ TDL model in [25] is built based on the similar hilly scenario, where a tap corresponds to a group of multipath components with similar propagation delays and gains as we modeled for our $2150 \mathrm{MHz}$ data set.

3.4.2. TDL Channel Model under Cutting 1. Table 5 deduces the TDL channel model under cutting 1 . It can be inferred that there are also several multipath signals; however, the LoS path plays a more dominant role in this scenario and the RMS delay spread is smaller than that of tunnel portal.

3.4.3. TDL Channel Model under Viaduct. Table 6 gives the TDL channel model parameters in the two subsections, viaduct near cutting 1 and viaduct near cutting 2 . We can observe that when the $\mathrm{Rx}$ is near the cutting 1 or cutting 2, a two- or three-ray model is applied. This is because the scattering and reflection of cutting 1 and cutting 2 contribute to the second and third paths. When the Rx is in the middle part of the viaduct, that is, far from the cutting 1 and cutting 2, there is only one LoS path which is dominant and almost no multipath component is detected.

3.4.4. TDL Channel Model under Cutting 2. As can be seen from Figure 6(f), only LoS path exists in cutting 2 area, because the $\mathrm{Rx}$ is far from the $\mathrm{Tx}$, the distances of the direct path and the reflection path from cutting 2 are substantially
TABLE 4: TDL channel model for tunnel portal.

\begin{tabular}{lccc}
\hline Scenario & Tap & Relative delay $(\mathrm{ns})$ & Average gain $(\mathrm{dB})$ \\
\hline \multirow{4}{*}{ Tunnel portal } & $950 \mathrm{MHz}$ & 0.0 \\
& 1 & 0 & -29.48 \\
& 2 & 119.7 & -31.84 \\
& 3 & 353.9 & -27.25 \\
& 5 & 452.0 & -25.14 \\
& 6 & 548.8 & -31.28 \\
\hline \multirow{5}{*}{ Tunnel portal } & 2 & 695.8 & \\
& 1 & $2150 \mathrm{MHz}$ & 0.0 \\
& 3 & 0 & -14.21 \\
& 4 & 174.1 & -18.23 \\
\hline
\end{tabular}

TABLE 5: TDL channel model for cutting.

\begin{tabular}{lccc}
\hline Scenario & Tap & Relative delay $(\mathrm{ns})$ & Average gain $(\mathrm{dB})$ \\
\hline & \multicolumn{3}{c}{$950 \mathrm{MHz}$} \\
& 1 & 0 & 0.0 \\
Cutting 1 & 2 & 82.9 & -36.86 \\
& 3 & 138.1 & -51.26 \\
& 4 & 192.6 & -52.93 \\
& 5 & 241.4 & -55.66 \\
\hline \multirow{5}{*}{ Cutting 1} & 3 & $2150 \mathrm{MHz}$ & \\
& 1 & 0 & 0.0 \\
& 2 & 95.7 & -32.16 \\
& 4 & 180.4 & -55.39 \\
& 5 & 254.7 & -57.35 \\
\hline
\end{tabular}

TABLE 6: TDL channel model for viaduct of both ends' connecting cuttings.

\begin{tabular}{lccc}
\hline Scenario & Tap & Relative delay $(\mathrm{ns})$ & Average gain $(\mathrm{dB})$ \\
\hline \multirow{3}{*}{ Viaduct near cutting 1 } & 1 & $050 \mathrm{MHz}$ & \\
& 2 & 108.3 & -37.27 \\
\hline \multirow{2}{*}{ Viaduct near cutting 2 } & 1 & 0 & 0.0 \\
& 2 & 89.5 & -9.5 \\
\hline & & $2150 \mathrm{MHz}$ & \\
& 1 & 0 & 0.0 \\
Viaduct near cutting 1 & 2 & 106.5 & -22.95 \\
& 3 & 428.4 & -39.6 \\
\hline \multirow{2}{*}{ Viaduct near cutting 2} & 1 & 0 & 0.0 \\
& 2 & 107.3 & -21.46 \\
\hline
\end{tabular}

equal, and the reflected signals from the remote mountain are quite weak.

\section{Scenario Reconstruction and RT Simulation}

4.1. Reconstruction of the Scenario. As a deterministic model, the propagation is greatly affected by the modeling of the 


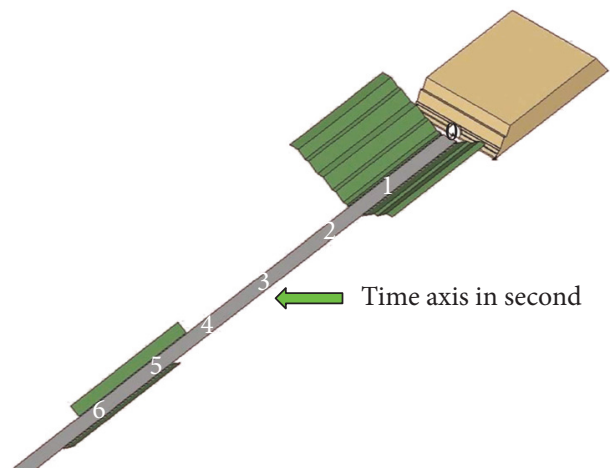

FIGURE 8: Reconstruction of the composite HSR scenario.

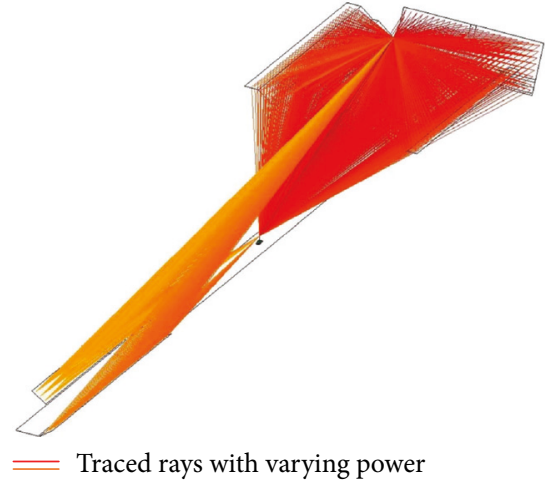

Figure 9: A snapshot of the RT simulation. scenario. As a result, precisely reconstructing the 3D propagation environment is the key factor for deterministic channel modeling. With the help of 3D modeling, the radio channel behavior of the composite scenario has been simulated by the deterministic propagation model. To begin with, the dimensions of the viaduct, cutting, and buildings are measured manually. Then, the latitude and longitude information of the test line is collected and the scenario around the test line is obtained by the satellite image of Google Earth. Finally, by gathering all the information, a complete 3D RT channel model library can be established.

In light of different radio propagation mechanisms, all the major objects in HSR scenario could be divided into the large-scale and the small-scale structures. The large-scale structures, including ballastless track, tunnel, cutting, and viaduct, are modeled as triangles and polygons with different electromagnetic parameters. The small-scale structures, including pylons and vegetation, are modeled as a conductive cylinder with a limited length. The scenario is composed of tunnel, mountain, cutting, viaduct, and ballastless track. It is essential to know the dielectric properties of materials for improving the accuracy of simulation. The reflection coefficients and scattering coefficients of all the materials above can be determined by the following approaches. First of all, the standardized parameters are defined in ITU-R recommendation, like ITU-R P.2040, ITU-R P.1411, ITU-R P.1238-7, and so on. These parameters can be set to the original inputs. Furthermore, for specific environments where materials are unavailable or insufficient in standards, measurements will be used to calibrate the coefficients. In this work, we apply the proposed approach in $[44,45]$, and the calibrated material parameters of cutting, viaduct, and others are implanted into the RT simulator after measurements. Thus, the RT simulator can be used for simulation to perform an in-depth analysis of the channel characteristics in specific scenario. Reconstruction of the composite HSR scenario for the RT simulation is shown in Figure 8.

4.2. 3D Ray-Optical Based Channel Simulation. The 3D rayoptical based channel simulation is used to establish 3D deterministic channel model. Several propagation mechanisms are taken into account to model the multipath nature of HSR channel. Firstly, the direct path between the $\mathrm{Tx}$ and $\mathrm{Rx}$ is calculated based on the distance and path loss. Secondly, specular reflection and diffuse scattering are calculated, with the bouncing order of reflection up to 2 for reducing the computational complexity. Furthermore, the contribution of diffraction is neglected because there is no obstacle between the Tx and Rx. Thus, the information of each ray can be obtained, including the type of path, path loss, path delay, and $3 \mathrm{D}$ angular properties. The positions of $\mathrm{Tx} / \mathrm{Rx}$, the directions of the antennas, and a snapshot of the RT result are illustrated in Figure 9. In this figure, the lines of different colors represent different received power level.

\section{Validation of RT Simulation and Further Analysis}

5.1. Validation of RT Simulation. For the purpose of improving RT algorithm efficiency, the objects involved in the deterministic channel model are dominant for the HSR, such as the viaduct, tunnel, and cutting. Hence, the model has excluded the objects that are remote, unobservable, and lower than the track. In RT algorithm, $\lambda / 16$ is taken as the sampling interval, where $\lambda$ refers to the wavelength. The other parameters used in the simulations are the same as those used in the actual measurements. Assuming that the train runs at $310 \mathrm{~km} / \mathrm{h}$, the time axis indicating the train location is illustrated in Figure 8.

Figure 10 shows the comparison of path losses between the measured and the simulated results. Due to the limitation of RT simulation, for example, the limited order of reflection, no diffraction mechanism modeling, and rough scene reconstruction, there is certain difference between RT simulated and measured path loss. However, the changing tendency is in agreement qualitatively at different frequency bands. Figure 11 shows the PDP based on the measurement data and the PDP predicted by the deterministic channel model in one snapshot for $950 \mathrm{MHz}$ and $2150 \mathrm{MHz}$. The prediction and measurement results are well consistent for the normalized power and multipath delay, except a few points (delay over $400 \mathrm{~ns}$ ) which do not match so well due to simplification of the remote scattering object. Indeed, the capability of capturing the dense multipath component (DMC) is the weakness of the RT simulation methods, which has been pointed out by numerical publication. In the scenario of this paper, the 
TABLE 7: Analysis of normalized small-scale fading.

\begin{tabular}{|c|c|c|c|c|c|}
\hline Zone index & 1 & 2 & 3 & 4 & 5 \\
\hline Time segment in s & $0 \sim 1.5$ & $1.5 \sim 2.7$ & $2.7 \sim 4.7$ & $4.7 \sim 5.6$ & $5.6 \sim 6.4$ \\
\hline Corresponding scenario & Tunnel portal and cutting & Viaduct & Viaduct & Joint of viaduct and cutting & Cutting \\
\hline Fading depth in $\mathrm{dB}(950 \mathrm{MHz})$ & 6.1 & 5.2 & 2.1 & 0.7 & 0.9 \\
\hline Maximum fading depth in $\mathrm{dB}(950 \mathrm{MHz})$ & 46.07 & 14.87 & 6.02 & 1.68 & 4.38 \\
\hline Fading depth in $\mathrm{dB}(2150 \mathrm{MHz})$ & 15.4 & 17.9 & 2.8 & 15.5 & 0.7 \\
\hline Maximum fading depth in $\mathrm{dB}(2150 \mathrm{MHz})$ & 48.37 & 37.87 & 3.35 & 59.04 & 1.37 \\
\hline
\end{tabular}

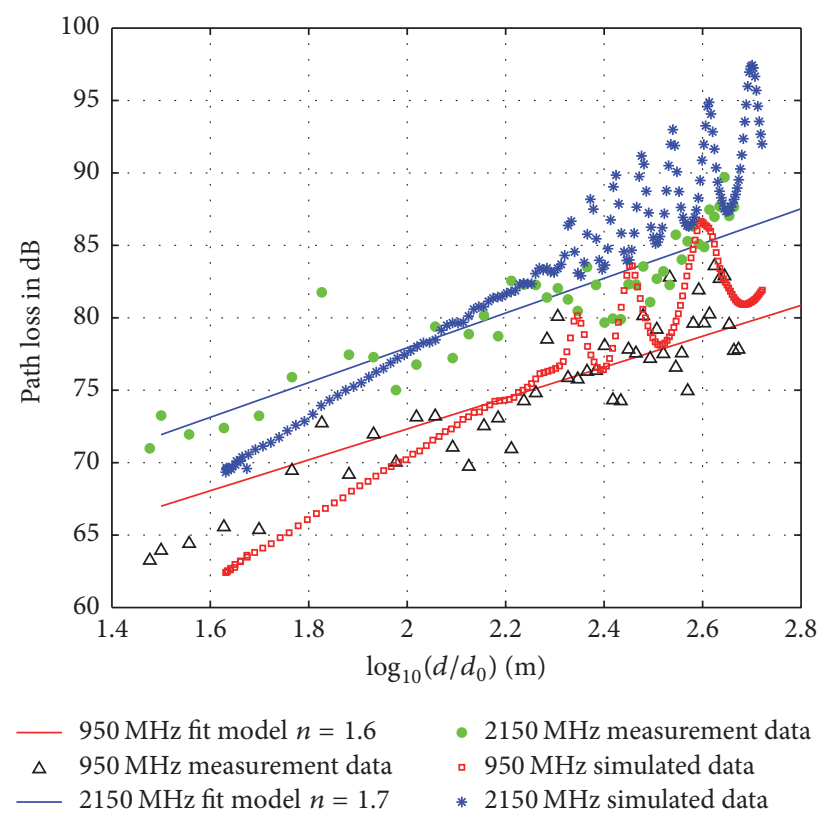

Figure 10: Comparison of path losses between the measured and the simulated results.

tunnel and cutting are typical closed or semiclosed environments, and, therefore, usually strong multipath components with extremely long delay are not expected. This is well reflected in the measurement data as well. Thus, for the target scenario, the RT simulator is applicative for further analysis.

5.2. Small-Scale Fading Analysis. Based on the above 3D scenario, small-scale fading characteristics are investigated through the channel simulation in this subsection. The signal bandwidth, power, antenna parameters, and location are the same as field measurement. Figure 12 illustrates the smallscale fading depth and maximum fading value as the function of time at $950 \mathrm{MHz}$ and $2150 \mathrm{MHz}$, respectively. Different time points reflect the different geographical locations in the composite scenario. The significantly different characteristics are found for small-scale fading in different locations which belong to different scenarios, given in Table 7 . It can be observed that the fading depth in zone 2 is greater than in zone 3 for both $950 \mathrm{MHz}$ and $2150 \mathrm{MHz}$, whereas the two zones are both viaduct region. The reason is that zone 2 is even closer to the cutting. In zone 4, the maximum fading depth for $2150 \mathrm{MHz}$ is about $-59 \mathrm{~dB}$, which is deeper than other zones, while the same phenomenon is not found so serious for $950 \mathrm{MHz}$. Similar results about the small-scale fading are obtained for the $900 \mathrm{MHz}$ data set in [43], but the maximum fading depth in [43] is lower than that in this paper. This result indicates that the composite scenario leads to more severe small-scale fading.

5.3. Stochastic Analysis. Wireless communication system must be developed for various propagation conditions, which motivates stochastic characteristic analysis that treats a linear time-varying channel as a random quantity. Since the channel has no great change in the stationarity interval which is assumed to be $10 \mathrm{~ms}$ [46], the hypothesis of wide-sense stationary uncorrelated scattering (WSSUS) is rational. According to WSSUS hypothesis in the stationary interval, the stochastic analysis is conducted subsequently.

5.4. PDP Properties Analysis. Based on the RT simulation, the PDP is studied in this subsection. Figure 13 gives PDP versus scenario time at two frequencies. In general, the changing trends and fluctuation from the deterministic model are similar to the measurements. The PDPs of $950 \mathrm{MHz}$ and $2150 \mathrm{MHz}$ are similar. When the train is near the transmitter position where the scenario is the tunnel portal and high cutting, more diffuse multipath components exist due to the rich scattering rays from tunnel external wall and the higher cutting. Dense diffuse multipaths are demonstrated; see Figure 13. The delay is mainly distributed in 0-200 ns, which is below the measured delay and the power of multipath is higher than the measurement data because of the simplification of the scenario.

5.5. Doppler Statistical Properties Analysis. Based on the RT simulation, Doppler statistical properties are analyzed in this subsection. The mean Doppler shift $\bar{\nu}$ is defined as

$$
\bar{v}=\frac{\int_{-\infty}^{+\infty} \nu P_{H}(\nu, f) d \nu}{\int_{-\infty}^{+\infty} P_{H}(\nu, f) d \nu},
$$

where $P_{H}$ is the Doppler power spectral density, $v$ is Doppler shift, and $f$ is frequency. The RMS Doppler spread $\sigma_{v}$ is defined as the square root of the second central moment of the Doppler power spectral density and is presented in (7), which can be used to characterize the time selectivity of the fading [41].

$$
\sigma_{v}=\sqrt{\overline{v^{2}}-(\bar{v})^{2}}
$$




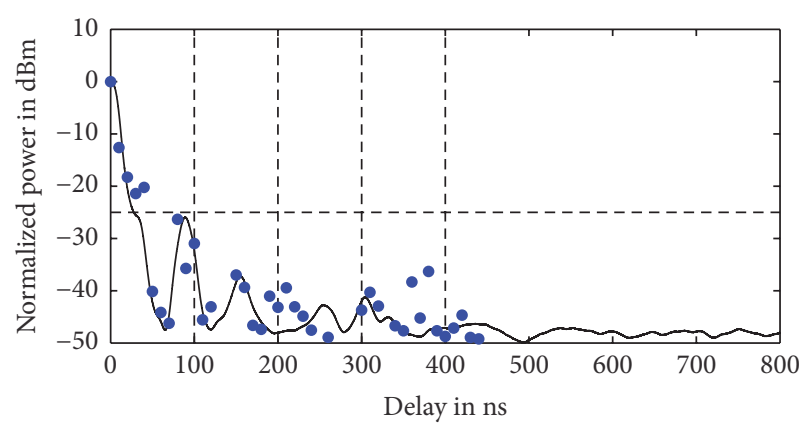

- Measured data

- Proposed deterministic model

(a) $950 \mathrm{MHz}$

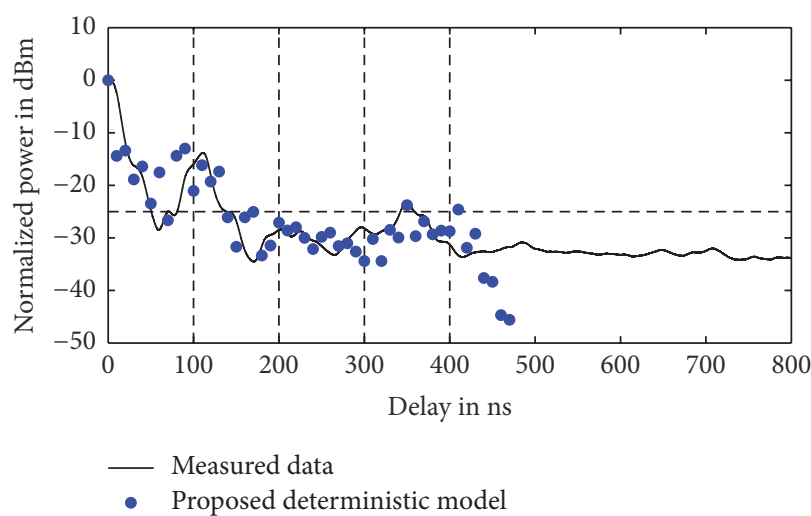

(b) $2150 \mathrm{MHz}$

FIGURE 11: Contrast of the PDP based on the measurement and the deterministic modeling.

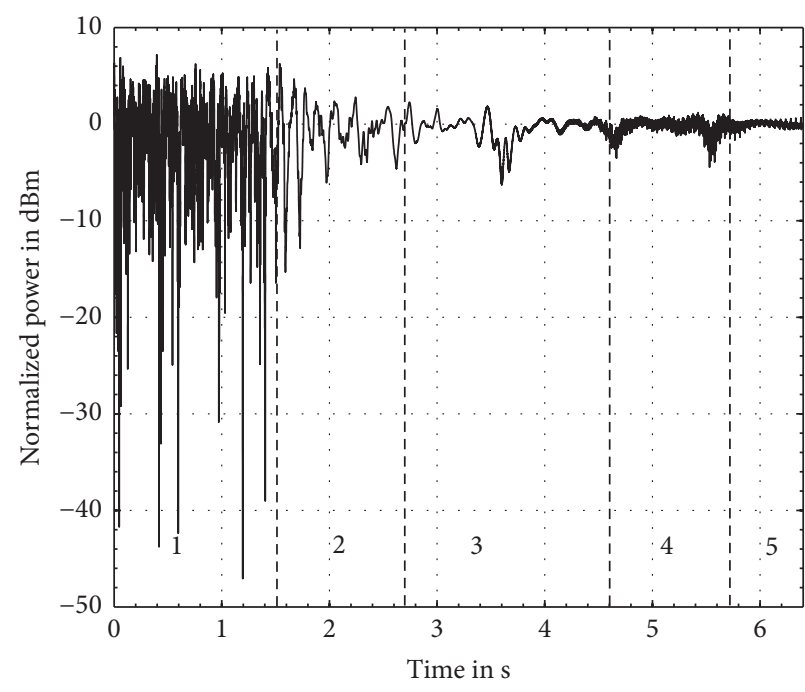

— Small-scale fading depth

(a) $950 \mathrm{MHz}$

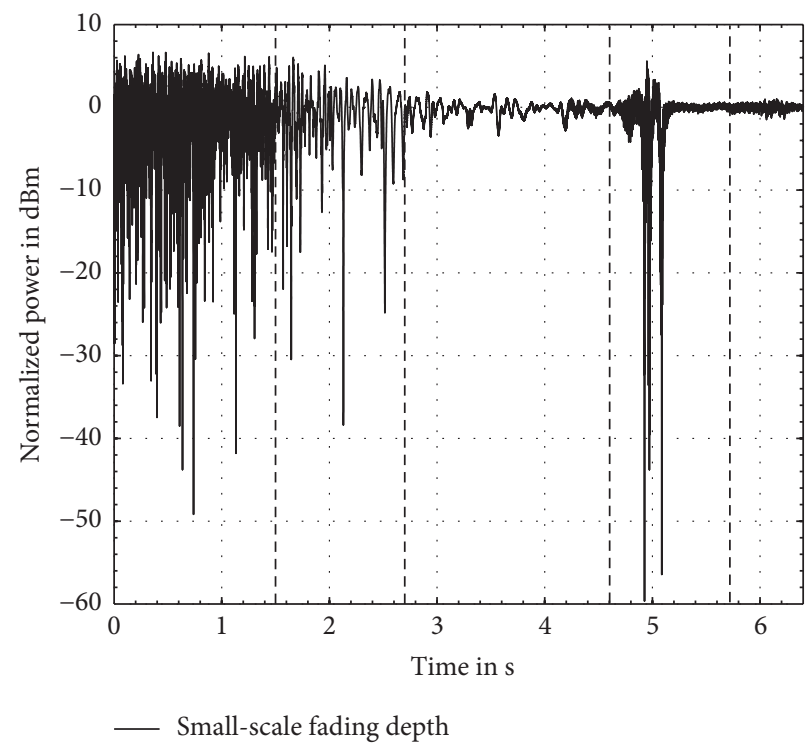

(b) $2150 \mathrm{MHz}$

FIGURE 12: Small-scale fading.

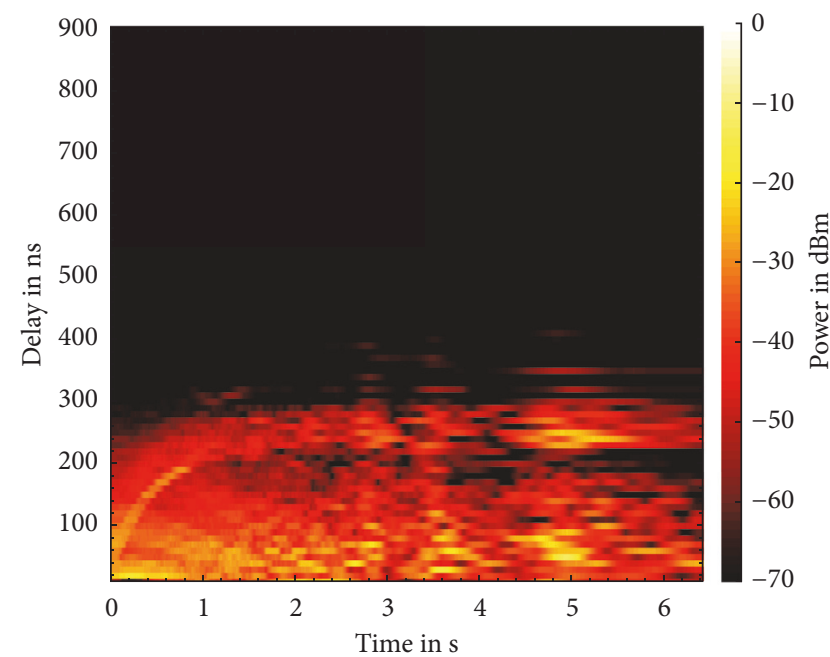

(a) $950 \mathrm{MHz}$

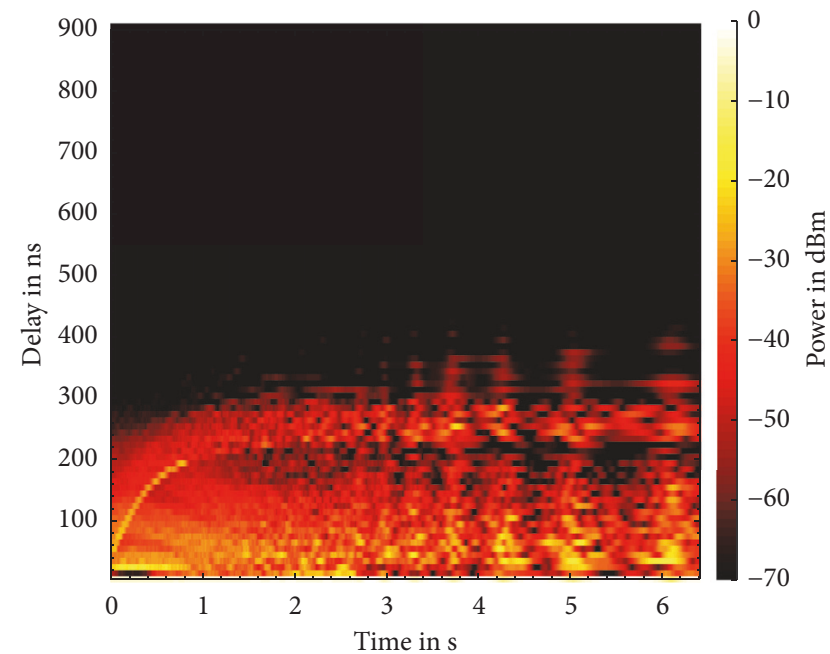

(b) $2150 \mathrm{MHz}$

FIGURE 13: PDP versus scenario time at different frequencies. 


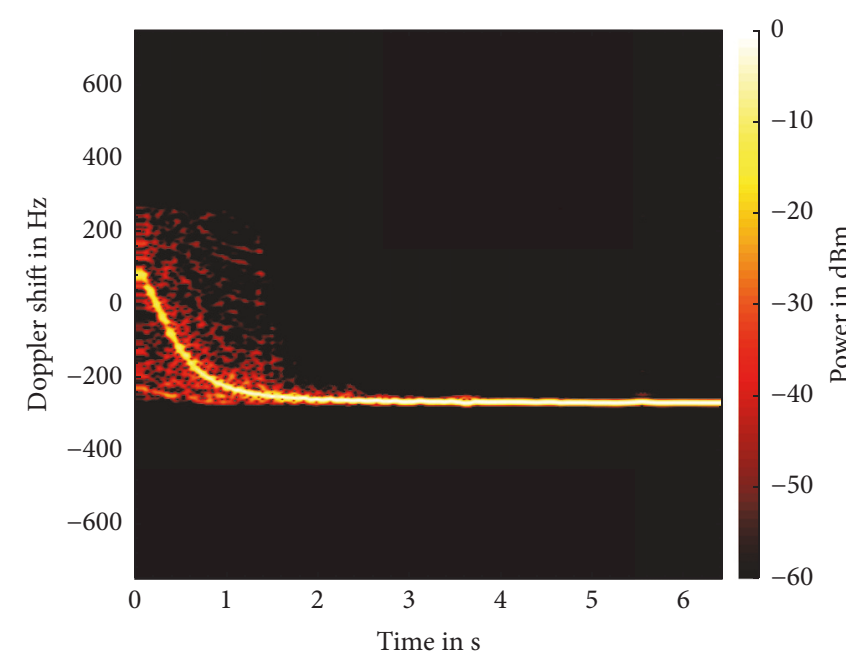

(a) $950 \mathrm{MHz}$

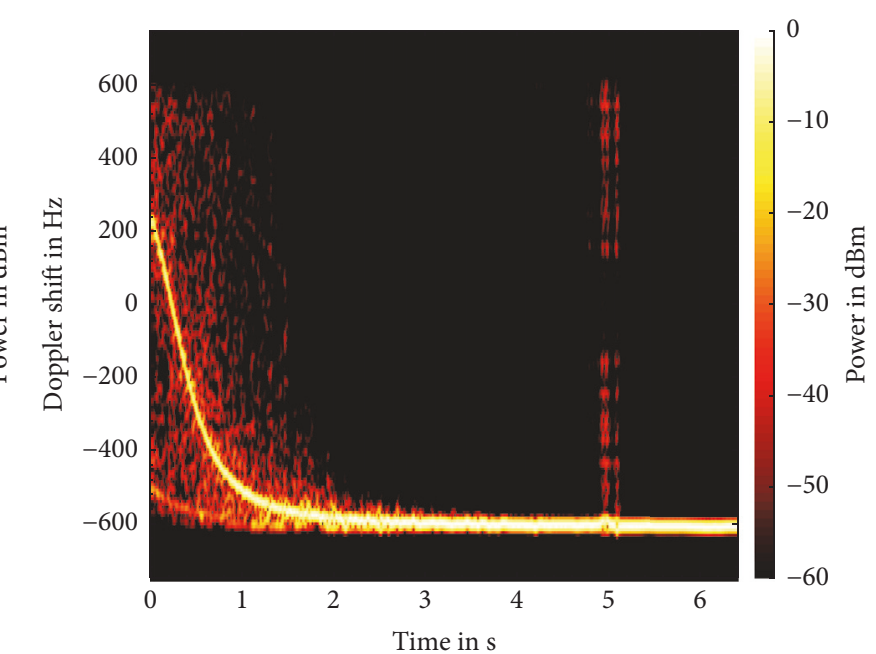

(b) $2150 \mathrm{MHz}$

FIGURE 14: Doppler power spectrum versus scenario time at different frequencies.

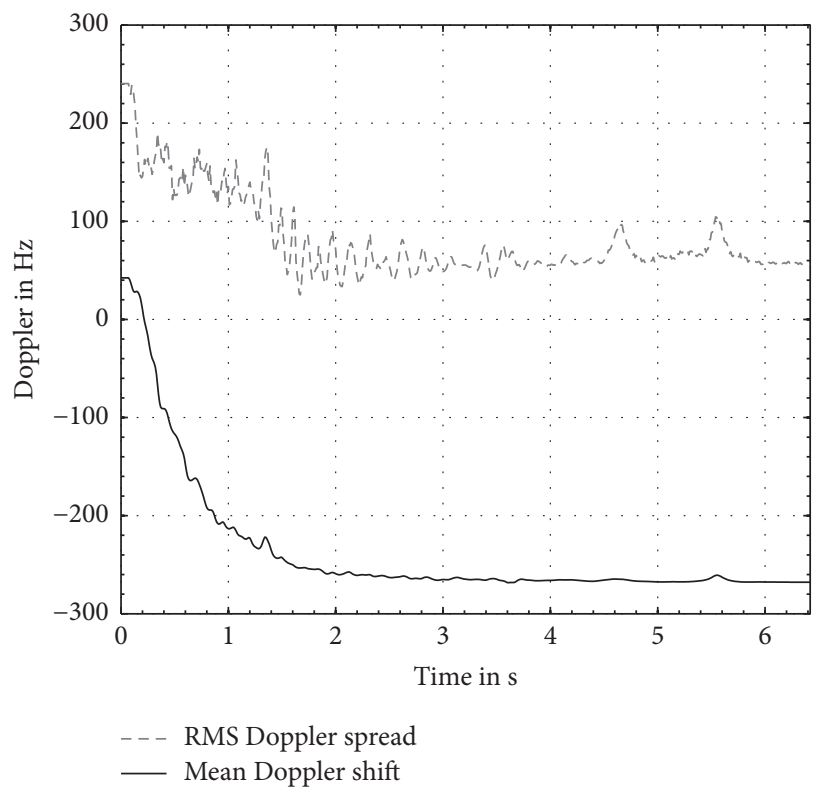

(a) $950 \mathrm{MHz}$

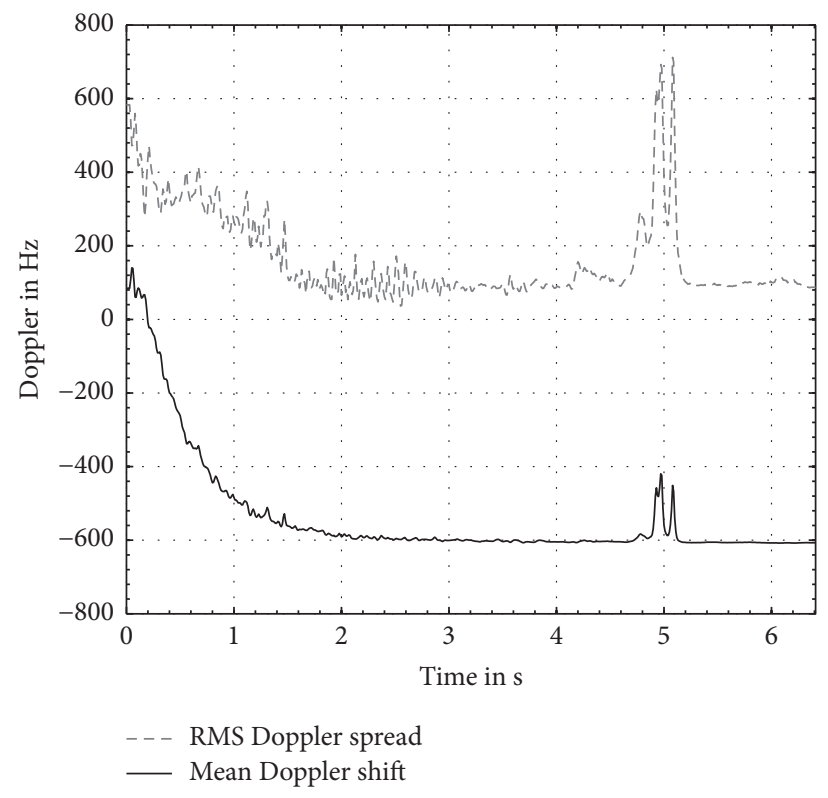

(b) $2150 \mathrm{MHz}$

FiguRE 15: RMS Doppler spread and mean Doppler shift versus scenario time at different frequencies.

The Doppler power spectrum, mean Doppler shift, and RMS Doppler spread of the two frequencies are given in Figures 14 and 15. When the train passes through the transmitter, the mean Doppler shift drifts drastically from $200 \mathrm{~Hz}$ to $-600 \mathrm{~Hz}$ for $2150 \mathrm{MHz}$ and from $100 \mathrm{~Hz}$ to $-260 \mathrm{~Hz}$ for $950 \mathrm{MHz}$. When the RMS Doppler spread is relatively large, the small-scale fading depth becomes larger, indicating that the dense scattering components from the cutting lead to severe Doppler spread. Moreover, if the delay spread is larger, the fading would be deeper. For $950 \mathrm{MHz}$, the Doppler spread is not so large as $2150 \mathrm{MHz}$ in simulations, so the serious fading does not occur. Therefore, the mobile station and base station should be designed to estimate the Doppler shift in real time and dynamically adjust the system parameters according to the Doppler shift.

5.6. The Correlation Coefficients between Delay and Doppler Domain. Based on the RT simulation, the cross-correlation coefficient between delay and Doppler domain is estimated in this subsection, which is defined as [47]

$$
\rho\left(\sigma_{\tau}, \sigma_{\nu}\right)=\frac{\sum_{k}^{M}\left(\sigma_{\tau}(k)-\overline{\sigma_{\tau}}\right)\left(\sigma_{\nu}(k)-\overline{\sigma_{\nu}}\right)}{\sqrt{\sum_{k}^{M}\left(\sigma_{\tau}(k)-\overline{\sigma_{\tau}}\right)^{2} \sum_{k}^{M}\left(\sigma_{\nu}(k)-\overline{\sigma_{\nu}}\right)^{2}}},
$$


TABLE 8: The correlation coefficients between delay and Doppler domain.

\begin{tabular}{lc}
\hline Scenarios & Correlation coefficients \\
\hline \multicolumn{2}{c}{$950 \mathrm{MHz}$} \\
Tunnel portal and cutting 1 & 0.5469 \\
Viaduct & 0.0308 \\
Cutting 2 & 0.1011 \\
\hline \multicolumn{2}{c}{$2150 \mathrm{MHz}$} \\
Tunnel portal and cutting 1 & 0.5082 \\
Viaduct & 0.0143 \\
Cutting 2 & 0.3032 \\
\hline
\end{tabular}

where $\sigma_{\tau}$ is delay spread, $\sigma_{\nu}$ is the Doppler spread, and $\overline{\sigma_{\tau}}$ and $\overline{\sigma_{\nu}}$ are the mean value of the data sets $\sigma_{\tau}(k)$ and $\sigma_{\nu}(k)$ with length $M$, respectively. The results of the extracted crosscorrelation coefficients are given in Table 8. There is a high correlation between the delay spread and the Doppler spread at the cutting area for different frequency bands. Meanwhile, the delay and Doppler spreads exhibit a low correlation when the train moves on the viaduct as sparse scatterers are on both track sides.

\section{Conclusion}

In this paper, the broadband wireless channel measurements using a customized channel sounder in a real HSR composite scenario at $950 \mathrm{MHz}$ and $2150 \mathrm{MHz}$ are reported and analyzed. The path loss exponent and parameters of time dispersion containing the number of paths, the mean excess delay, and the RMS delay spread are estimated. The TDL channel model is proposed for the HSR composite scenarios containing tunnel portal, cutting, and viaduct based on the actual measurement data. The analysis reveals some important phenomena for the composite scenario: the difference of RMS delay at two frequencies is very small while the propagation loss is more serious; the cutting and tunnel portal will bring greater RMS delay; the multipath in viaduct is more influenced by the two cuttings that are connected to the viaduct, because the interference from the reflection and scattering of the cuttings produces not only deep fading, but also Doppler dispersion and delay spread. The deterministic channel model of the composite scenario, which is reconstructed using the 3D RT method, is put forward in this paper. The channel model is compared and validated with measurement data. The transition regions of different scenarios could be clearly identified by the normalized small-scale fading and Doppler characteristics analysis. Our future work will continue to analyze cluster birth and death behavior of the multipath components in this scenario. As radio channels have profound impacts on the field strength coverage, reliability, and quality of service of HSR mobile communication system, the results are useful for providing guidance on the broadband HSR communication system design, network planning, and optimization.

\section{Conflicts of Interest}

The authors declare that there are no conflicts of interest regarding the publication of this paper.

\section{Acknowledgments}

The authors express their thanks to the support in part from the National Key R\&D Program of China under Grant no. 2016YFB1200102-04, the National Natural Science Foundation of China under Grants 61501021, U1334202, 61501020, and 61771037, the Project of China Railway Corporation under Grants 2016X009-E, 2016X003-L, and 2016X003-O, the Fundamental Research Funds for the Central Universities under Grant 2016JBM076, State Key Lab of Rail Traffic Control and Safety Project under Grant RCS2016ZJ005, and Beijing Natural Science Foundation under Grant L161009.

\section{References}

[1] L. Lei, J. Lu, Y. Jiang et al., "Stochastic delay analysis for train control services in next-generation high-speed railway communications system," IEEE Transactions on Intelligent Transportation Systems, vol. 17, no. 1, pp. 48-64, 2016.

[2] C. Briso-Rodríguez, C. F. López, J. R. O. Fernández et al., "Broadband access in complex environments: LTE on railway," IEICE Transactions on Communications, vol. E97-B, no. 8, pp. 1514-1527, 2014.

[3] Y.-S. Song, J. Kim, S. W. Choi, and Y.-K. Kim, "Long term evolution for wireless railway communications:testbed deployment and performance evaluation," IEEE Communications Magazine, vol. 54, no. 2, pp. 138-145, 2016.

[4] J. Calle-Sanchez, M. Molina-Garcia, and J. I. Alonso, “Top challenges of LTE to become the next generation railway communication system," in Proceedings of the COMPRAIL 2012. 13th International Conference on Design and Operation in Railway Engineering, pp. 85-96, Wessex Institue of Technology, New Forest, UK, September 2012.

[5] L. Brunel, H. Bonneville, and A. Charaf, "Throughput performance of 3GPP LTE system in railway environment," in Communication Technologies for Vehicles, vol. 10222 of Lecture Notes in Computer Science, pp. 60-71, Springer International Publishing, Cham, Switzerland, 2017.

[6] R. He, B. Ai, G. Wang et al., "High-Speed railway communications: from GSM-R to LTE-R," IEEE Vehicular Technology Magazine, vol. 11, no. 3, pp. 49-58, 2016.

[7] J. Calle-Sánchez, M. Molina-García, J. I. Alonso, and A. Fernández-Durán, "Long term evolution in high speed railway environments: feasibility and challenges," Bell Labs Technical Journal, vol. 18, no. 2, pp. 237-253, 2013.

[8] H. Bonneville, L. Brunel, and D. Mottier, "Standardisation roadmap for next train radio telecommunication systems," Lecture Notes in Computer Science (including subseries Lecture Notes in Artificial Intelligence and Lecture Notes in Bioinformatics): Preface, vol. 9669, pp. 51-61, 2016.

[9] 3rd Generation Partnership Project, "Technical specification group radio access network; evolved universal terrestrial radio access (E-UTRA); base station (BS) radio transmission and reception," Tech. Rep., 3GPP, Sophia Antipolis, France, 2016, TS 36.104 V14.0.0. 
[10] M. Zhao, M. Wu, Y. Sun et al., "Analysis and modeling of the LTE broadband channel for train-ground communications on highspeed railway," in Proceedings of the 2013 IEEE 78th Vehicular Technology Conference, VTC Fall 2013, USA, September 2013.

[11] K. Guan, Z. Zhong, B. Ai, and T. Kurner, "Empirical models for extra propagation loss of train stations on high-speed railway," IEEE Transactions on Antennas and Propagation, vol. 62, no. 3, pp. 1395-1408, 2014.

[12] T. Zhou, C. Tao, S. Salous, L. Liu, and Z. Tan, "Channel characterization in high-speed railway station environments at 1.89 GHz," Radio Science, vol. 50, no. 11, pp. 1176-1186, 2015.

[13] K. Guan, Z. Zhong, B. Ai, and T. Kurner, "Propagation measurements and analysis for train stations of high-speed railway at 930 MHz," IEEE Transactions on Vehicular Technology, vol. 63, no. 8, pp. 3499-3516, 2014.

[14] T. Zhou, C. Tao, L. Liu, and Z. Tan, "A semiempirical MIMO channel model in obstructed viaduct scenarios on high-speed railway," International Journal of Antennas and Propagation, vol. 2014, Article ID 287159, 10 pages, 2014.

[15] R. He, Z. Zhong, B. Ai, G. Wang, J. Ding, and A. F. Molisch, "Measurements and analysis of propagation channels in highspeed railway viaducts," IEEE Transactions on Wireless Communications, vol. 12, no. 2, pp. 794-805, 2013.

[16] R. Zhao, M. Wu, X. Xiang, and J. Yang, "Measurement and modeling of the LTE train-ground channel for high-speed railway in viaduct scenario," in Proceedings of the 80th IEEE Vehicular Technology Conference, VTC 2014-Fall, Canada, September 2014.

[17] R. He, Z. Zhong, B. Ai, and J. Ding, "An empirical path loss model and fading analysis for high-speed railway viaduct scenarios," IEEE Antennas and Wireless Propagation Letters, vol. 10, pp. 808-812, 2011.

[18] R. He, Z. Zhong, B. Ai, J. Ding, Y. Yang, and A. F. Molisch, "Short-term fading behavior in high-speed railway cutting scenario: Measurements, analysis, and statistical models," IEEE Transactions on Antennas and Propagation, vol. 61, no. 4, pp. 2209-2222, 2013.

[19] J.-H. Qiu, C. Tao, L. Liu, and Z.-H. Tan, "Research on measurement and modeling of wireless channel multipath propagation properties for U-shape cutting," Tiedao Xuebao/Journal of the China Railway Society, vol. 36, no. 1, pp. 40-48, 2014.

[20] K. Guan, Z. Zhong, B. Ai, and T. Kürner, "Propagation measurements and modeling of crossing bridges on high-speed railway at $930 \mathrm{MHz}$," IEEE Transactions on Vehicular Technology, vol. 63 , no. 2, pp. 502-517, 2014.

[21] C. Briso-Rodriguez, J. M. Cruz, and J. I. Alonso, "Measurements and modeling of distributed antenna systems in railway tunnels," IEEE Transactions on Vehicular Technology, vol. 56, no. 5, pp. 2870-2879, 2007.

[22] J. Alonso, S. Capdevila, B. Izquierdo, and J. Romeu, "Propagation measurements and simulations in tunnel environment at 5.8GHz," in Proceedings of the 2008 IEEE International Symposium on Antennas and Propagation and USNC/URSI National Radio Science Meeting, APSURSI, USA, July 2008.

[23] K. Guan, Z. D. Zhong, J. I. Alonso, and C. Briso-Rodríguez, "Measurement of distributed antenna systems at $2.4 \mathrm{GHz}$ in a realistic subway tunnel environment," IEEE Transactions on Vehicular Technology, vol. 61, no. 2, pp. 834-837, 2012.

[24] J.-M. Molina-Garcia-Pardo, J.-V. Rodríguez, and L. Juan-Llácer, "Wide-band measurements and characterization at $2.1 \mathrm{GHz}$ while entering in a small tunnel," IEEE Transactions on Vehicular Technology, vol. 53, no. 6, pp. 1794-1799, 2004.
[25] Y. Zhang, Z. He, W. Zhang, L. Xiao, and S. Zhou, "Measurementbased delay and doppler characterizations for high-speed railway hilly scenario," International Journal of Antennas \& Propagation, vol. 2014, no. 4, pp. 1-8, 2014.

[26] L. Liu, C. Tao, J. Qiu et al., "Position-based modeling for wireless channel on high-speed railway under a viaduct at $2.35 \mathrm{GHz}$," IEEE Journal on Selected Areas in Communications, vol. 30, no. 4, pp. 834-845, 2012.

[27] Y. Yang and P. Fan, "Doppler frequency offset estimation and diversity reception scheme of high-speed railway with multiple antennas on separated carriage," Journal of Modern Transportation, vol. 20, no. 4, pp. 227-233, 2012.

[28] K. Guan, Z. Zhong, B. Ai, and T. Kürner, "Deterministic propagation modeling for the realistic high-speed railway environment," in Proceedings of the 2013 IEEE 77th Vehicular Technology Conference, VTC Spring 2013, deu, June 2013.

[29] J. Yang, B. Ai, K. Guan et al., "Deterministic modeling and stochastic analysis for channel in composite high-speed railway scenario," in Proceedings of the 83rd IEEE Vehicular Technology Conference, VTC Spring 2016, China, May 2016.

[30] X. Yin, Y. Ji, and H. Yan, "Measurement-based characterization of $15 \mathrm{GHz}$ propagation channels in a laboratory environment," IEEE Access, vol. 5, pp. 1428-1438, 2017.

[31] D. He, G. Liang, J. Portilla, and T. Riesgo, "A novel method for radio propagation simulation based on automatic $3 \mathrm{D}$ environment reconstruction," Radioengineering, vol. 21, no. 4, pp. 985992, 2012.

[32] J. Nuckelt, T. Abbas, F. Tufvesson, C. Mecklenbräuker, L. Bernadó, and T. Kürner, "Comparison of ray tracing and channel-sounder measurements for vehicular communications," in Proceedings of the 2013 IEEE 77th Vehicular Technology Conference, VTC Spring 2013, Germany, June 2013.

[33] L. Zhang, Channel measurement and modeling in complex environments [Ph.D. thesis], Technical University of Madrid, Madrid, Spain, 2016.

[34] S. Piersanti, L. A. Annoni, and D. Cassioli, "Millimeter waves channel measurements and path loss models," in Proceedings of the 2012 IEEE International Conference on Communications, ICC 2012, pp. 4552-4556, Canada, June 2012.

[35] S. Sun, T. S. Rappaport, T. A. Thomas et al., "Investigation of Prediction Accuracy, Sensitivity, and Parameter Stability of Large-Scale Propagation Path Loss Models for 5G Wireless Communications," IEEE Transactions on Vehicular Technology, vol. 65, no. 5, pp. 2843-2860, 2016.

[36] M. K. Samimi and T. S. Rappaport, "3-D millimeter-wave statistical channel model for $5 \mathrm{G}$ wireless system design," IEEE Transactions on Microwave Theory and Techniques, vol. 64, no. 7, pp. 2207-2225, 2016.

[37] P. Liu, A. Bo, D. W. Matolak, J.-W. Ding, R. Sun, and Y. Li, "Wave-guide effects in urban viaduct propagation channels for high-speed railway at $930 \mathrm{MHz}$," in Proceedings of the 8th European Conference on Antennas and Propagation, EuCAP 2014, pp. 3479-3483, Netherlands, April 2014.

[38] I. Sen and D. W. Matolak, "Vehicle-vehicle channel models for the 5-GHz band," IEEE Transactions on Intelligent Transportation Systems, vol. 9, no. 2, pp. 235-245, 2008.

[39] R. He, A. F. Molisch, F. Tufvesson, Z. Zhong, B. Ai, and T. Zhang, "Vehicle-to-vehicle propagation models with large vehicle obstructions," IEEE Transactions on Intelligent Transportation Systems, vol. 15, no. 5, pp. 2237-2248, 2014.

[40] L. Zhang, C. Briso, J. R. O. Fernandez et al., "Delay spread and electromagnetic reverberation in subway tunnels and stations," 
IEEE Antennas and Wireless Propagation Letters, vol. 15, pp. 585588, 2016.

[41] A. F. Molisch and M. Steinbauer, "Condensed parameters for characterizing wideband mobile radio channels," International Journal of Wireless Information Networks, vol. 6, no. 3, pp. 133154, 1999.

[42] M. S. Varela and M. G. Sánchez, "RMS delay and coherence bandwidth measurements in indoor radio channels in the UHF band," IEEE Transactions on Vehicular Technology, vol. 50, no. 2, pp. 515-525, 2001.

[43] B. Chen, Z. Zhong, B. Ai, K. Guan, R. He, and D. G. Michelson, "Channel characteristics in high-speed railway: a survey of channel propagation properties," IEEE Vehicular Technology Magazine, vol. 10, no. 2, pp. 67-78, 2015.

[44] T. Abbas, J. Nuckelt, T. Zemen, C. F. Mecklenbräuker, and F. Tufvesson, "Simulation and measurement-based vehicle-tovehicle channel characterization: accuracy and constraint analysis," IEEE Transactions on Antennas and Propagation, vol. 63, no. 7, pp. 3208-3218, 2015.

[45] D. He, K. Guan, A. Fricke et al., "Stochastic channel modeling for kiosk applications in the terahertz band," IEEE Transactions on Terahertz Science \& Technology, vol. PP, no. 99, pp. 1-12, 2017.

[46] A. Ghazal, C.-X. Wang, H. Haas et al., "A non-stationary geometry-based stochastic model for MIMO high-speed train channels," in Proceedings of the 2012 12th International Conference on ITS Telecommunications, ITST 2012, pp. 7-11, Taiwan, November 2012.

[47] M. Zhu, G. Eriksson, and F. Tufvesson, “The COST 2100 channel model: parameterization and validation based on outdoor MIMO measurements at $300 \mathrm{MHz}$, IEEE Transactions on Wireless Communications, vol. 12, no. 2, pp. 888-897, 2013. 


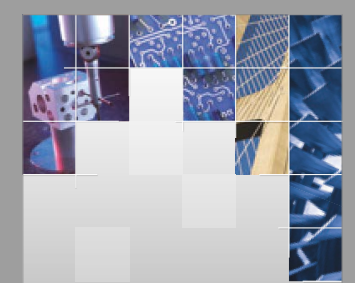

\section{Enfincering}
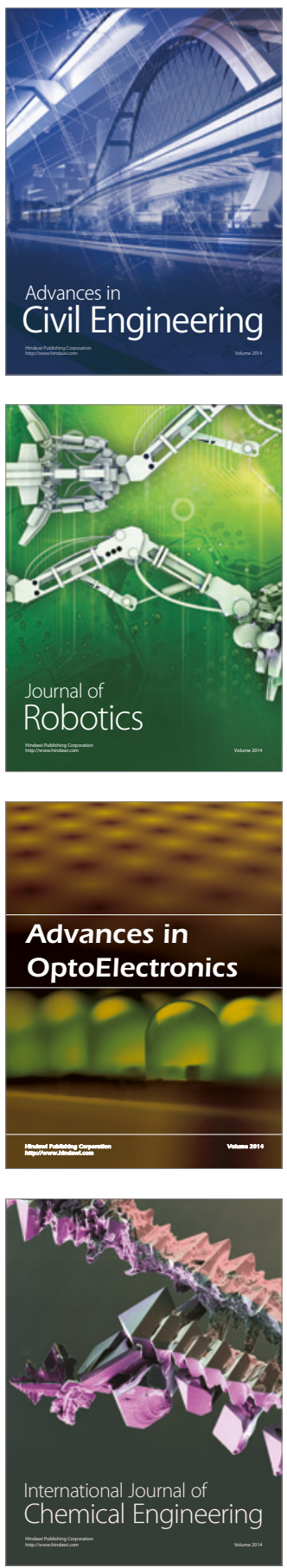

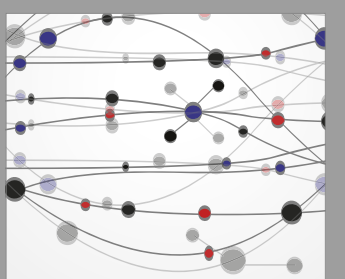

The Scientific World Journal

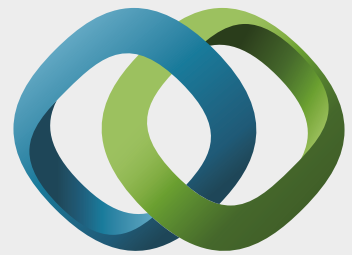

\section{Hindawi}

Submit your manuscripts at

https://www.hindawi.com
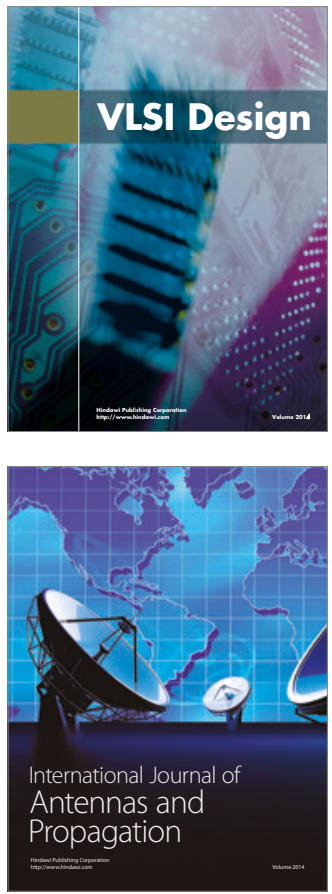

\section{Rotating}

Machinery
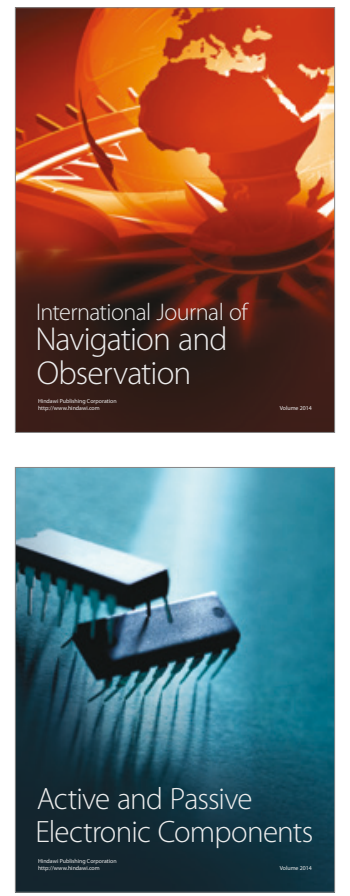
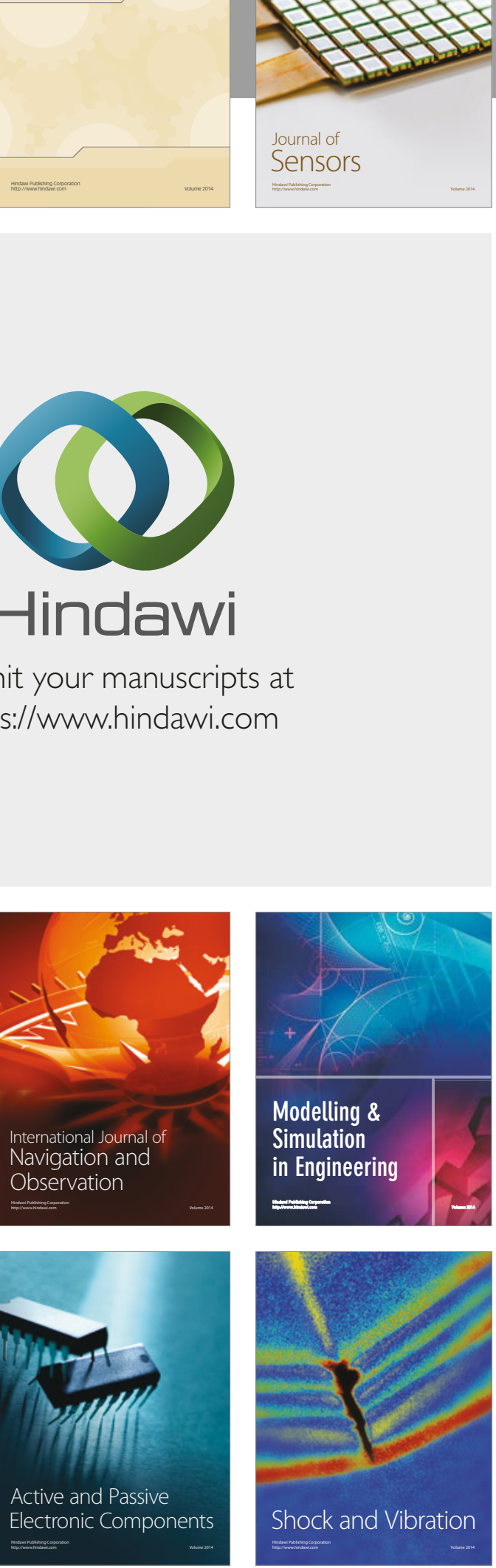
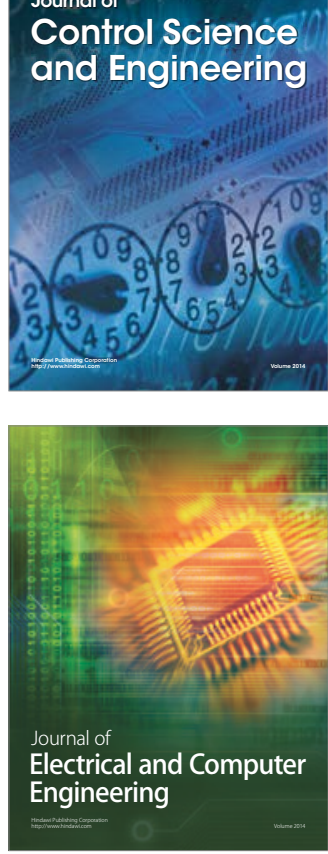

Distributed

Journal of

Control Science

and Engineering
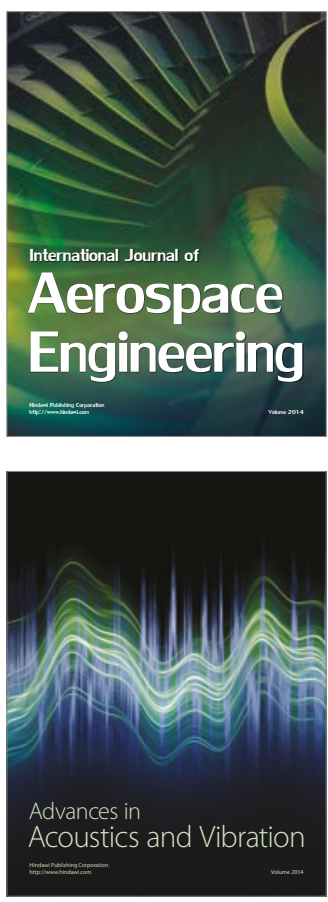

Sensor Networks 